\title{
Antimicrobial, Antioxidant and Cytotoxic Activity of Streptomyces species from Western Ghat Soils of Karnataka, India
}

\author{
Prashith Kekuda T. ${ }^{1}$, Onkarappa $\mathbf{R}^{1^{*}}$, Gautham S.A ${ }^{1}$, Sunita C. Mesta ${ }^{1}$ \\ and Raghavendra H.L ${ }^{2}$
}

\author{
${ }^{1}$ Department of Microbiology, Sahyadri Science College (Autonomous), Shivamogga-01, Karnataka, India \\ ${ }^{2}$ School of Medicine, College of Medical and Health Sciences, Wollega University, Post Box No: 395, \\ Nekemte, Ethiopia
}

\begin{tabular}{|c|c|}
\hline \multirow{4}{*}{$\begin{array}{l}\text { Abstract } \\
\text { Western Ghats of India represents one of the global biodiversity hotspots. The present study } \\
\text { was carried out to isolate and determine antimicrobial, cytotoxic and antioxidant activity of } \\
\text { actinomycetes from soils of different regions of Western Ghats of Shivamogga district, } \\
\text { Karnataka, India. A total of } 182 \text { isolates were recovered from } 57 \text { soil samples collected at } 13 \\
\text { different regions of Western Ghat region of Shivamogga district by serial dilution-plating } \\
\text { technique. Out of } 182 \text { isolates, } 17 \text { isolates were chosen for solvent extraction and to } \\
\text { determine antimicrobial, cytotoxic and antioxidant activity. Solvent extracts of these isolates } \\
\text { exhibited varied antimicrobial potency against Gram positive and Gram negative bacteria, } \\
\text { yeasts and molds. Ethyl acetate was found to be best solvent for extraction of bioactive } \\
\text { metabolites from most of isolates followed by butanol and hexane. Cytotoxic activity, as } \\
\text { evaluated by brine shrimp lethality bioassay, revealed the potency of extracts to cause dose } \\
\text { dependent mortality of brine shrimp larvae. Solvent extracts exhibited dose dependent } \\
\text { antioxidant activity, as evaluated by DPPH, ABTS, Metal chelating and Ferric reducing } \\
\text { assays. Western Ghat actinomycetes appear to be promising resources of bioactive agents } \\
\text { and can be exploited industrially. Further studies on recovery of active constituents from } \\
\text { solvent extracts and their bioactivity determinations are to be carried out. }\end{array}$} & Article Information \\
\hline & $\begin{array}{l}\text { Article History: } \\
\text { Received : }: 21-02-2015 \\
\text { Revised : } 13-06-2015 \\
\text { Accepted : } 15-06-2015\end{array}$ \\
\hline & $\begin{array}{l}\text { Keywords: } \\
\text { Actinomycetes } \\
\text { Streptomyces } \\
\text { Western Ghats } \\
\text { Antimicrobial }\end{array}$ \\
\hline & $\begin{array}{l}{ }^{*} \text { Corresponding Author: } \\
\text { R. Onkarappa } \\
\text { E-mail: } \\
\text { onkarappa.r@gmail.com }\end{array}$ \\
\hline
\end{tabular}

\section{INTRODUCTION}

Infectious diseases are the world's leading cause of premature deaths. Bacteria, viruses, fungi and other parasites cause a huge number of diseases in humans and these diseases are due to a complex interaction between the pathogen, host and the environment. The discovery of antibiotics and their subsequent use had eradicated these infections which once threatened mankind. These antibiotics saved countless lives. However, the indiscriminate use of these antimicrobial drugs led to the development of multidrug resistant pathogens. Drug resistance is a serious challenge to the field of medicine. Moreover, the pathogens have the ability to acquire and transmit the resistance genes which has made the situation ever worst. Many antibiotics such as vancomycin, methicillin, penicillin, fluconazole etc., are no longer effective against many pathogens. This has necessitated a search for new antimicrobials (Parea and Patterson, 2002; Davies and Davies, 2010; Pallavi et al., 2013; Kekuda et al., 2013a).

Like infectious diseases, cancer is another serious clinical problem posing a significant social and economic impact on the health care system. Cancer is the second largest cause of death in the world and it affects $>200$ cell types. The major characteristic of cancer is the lack of control of the cell proliferation, differentiation and death, invading organs and tissues. Cancer affects millions of patients worldwide even after advancements in diagnosis, prevention and therapy and reduces the quality of life. One of the most widely used approaches for treatment of cancer is chemotherapy. Drugs have been used for the treatment of various kinds of cancers. However, development of resistance to chemotherapeutic drugs affects the effective killing of the cancer cells, resulting in tumor recurrence. Besides, the patients may also suffer from side-effects such as cardiac and other toxicities. Hence, finding newer drugs effective against cancer cells is a major portion of current pharmacological research (de Mesquita et al., 2009; Yaacob et al., 2010; Kumar et al., 2011).

Living tissues derive energy from aerobic metabolism. However, these organisms are under threat of oxidative damage induced by reactive oxygen species such as superoxide, hydroxyl, peroxyl and alkoxyl radicals, hydrogen peroxide and singlet oxygen. Free radicals possess single unpaired electron and are known to be highly reactive against biologically important macromolecules viz., carbohydrates, proteins, lipids and nucleic acids. These oxygen species play an important 
role related to the degenerative or pathological processes of various serious diseases such as aging, cancer, coronary heart disease, Alzheimer's disease, neurodegenerative disorders, atherosclerosis, cataracts and inflammation. To counteract the threats posed by these highly reactive species, cells are equipped with antioxidant defense systems which include enzymes and antioxidant molecules. However, during pathophysiological conditions, there is an extra need for antioxidants. Synthetic antioxidants such as BHT, BHA and $P G$ are suspected to have toxic or carcinogenic effects. Hence, searching for antioxidants from natural sources such as plants and microbes is of much interest (Teow et al., 2007; Chang et al., 2007; Ebrahimzadeh et al., 2010; Aiyegoro and Okoh, 2010; Kekuda et al., 2013a)

Actinomycetes (order Actinomycetales) are Gram positive bacteria characterized by the formation of substrate and aerial mycelium, presence of spores and a high GC content. Considerable attention has been paid to this group notably after the discovery of streptomycin. Actinomycetes are ubiquitous in distribution and are physiologically diverse bacteria. They are responsible for characteristic earthy odor of soil. They are biotechnologically versatile prokaryotes and produce a number of extracellular enzymes and other bioactive agents, notably antibiotics. The biodegradation ability of these actinomycetes is extremely significant in recycling of materials in nature. Often, these organisms are involved in the degradation of harmful chemicals such as pesticides and herbicides. Besides, actinomycetes are significant in agriculture in terms of their biocontrol and nitrogen fixing ability (Kim et al., 1998; Gautham, 2012; Kekuda et al., 2010a; Kekuda and Onkarappa, 2015).

The genus Streptomyces constitute the major actinomycete genera. The species of Streptomyces are filamentous, aerobic spore formers possessing rich GC content $(69-73 \%)$ in their genome. They form extensive branching substrate and aerial mycelia. The morphological differentiation of Streptomyces involves the formation of hyphae that differentiate into a chain of spores. Streptomyces are ubiquitous in soil and contribute significantly to the turnover of complex biopolymers such as cellulose, lignin, pectin, hemicellulose, keratin and chitin. The species of Streptomyces have produced $>75 \%$ bioactive metabolites, mainly antibiotics. These metabolites have found medicinal (human and veterinary) and agricultural importance and are known to exhibit a range of bioactivities (Kokare et al., 2004; Yadav et al., 2008; ; Kekuda et al., 2010a; Priya et al., 2012; Procopio et al., 2012).

Western Ghats of India are considered as one of the global biodiversity hotspots. Although Western Ghats cover an area of $1,80,000 \mathrm{~km}^{2}$ which is just under $6 \%$ of the land area of India, they contain $>30 \%$ of all plant, fish, herpeto-fauna, birds, and mammal species found in India. The hill range runs through Gujarat, Maharashtra, Goa, Karnataka and Kerala. The Western Ghats represents the 'gene pool' harboring numerous species of plants, animals and microbes. The unique biodiversity of Western Ghats are protected and conserved by biosphere reserves, national parks and wildlife sanctuaries (Gautham, 2012; Nampoothiri et al., 2013). Microbiological studies on soils of various places of Western Ghats of Karnataka such as Thirthahalli, Agumbe, Talakaveri, Kodachadri and
Kudremukh have been carried out. It has been shown that Western Ghat actinomycetes possess bioactivities such as antimicrobial, antioxidant, enzyme inhibitory, insecticidal, cytotoxic, anthelmintic, analgesic, antiinflammatory, CNS depressant and antipyretic activities (Kekuda et al., 2010b; Shobha and Onkarappa, 2011; Gautham et al., 2012; Manasa et al., 2012; Kekuda et al., 2012; Kekuda et al., 2013a; Kekuda et al., 2013b; Gautham et al., 2013; Junaid et al., 2013; Kekuda and Onkarappa, 2014a; Kekuda et al., 2014b; Kekuda and Onkarappa, 2015). The present study was conducted to determine antimicrobial, antioxidant and cytotoxic efficacy of solvent extracts from actinomycetes recovered from soils of different regions of Western Ghats of Shivamogga district, Karnataka, India.

\section{MATERIALS AND METHODS}

\section{Collection of Soil Samples}

A total of 57 rhizosphere soil samples were collected from 13 places of Western Ghats of Shivamogga district, Karnataka. The surface soil was removed to eliminate leaf litter and other extraneous matter. The soil samples were collected from a depth of $5-10 \mathrm{~cm}$ in sterile plastic pouches. The samples were brought to the laboratory and dried at $40^{\circ} \mathrm{C}$ under aseptic conditions (Kekuda et al., 2013a). The places of soil collection and the number of soil samples collected is shown in Table 1.

\section{Isolation of Actinomycetes}

Serial dilution-plating method was employed to isolate actinomycetes from soils samples. Soil samples were serially diluted up to $10^{-7}$ followed by plating (by spread plate method) on sterile Starch casein nitrate (SCN) agar plates. The plates were incubated aerobically at $20^{\circ} \mathrm{C}$ for up to 14 days. Well isolated colonies showing typical characteristics of actinomycetes were subcultured and maintained on SCN agar slants. The medium was supplemented with fluconazole in order to prevent fungal contamination (Aneja, 1996).

\section{Primary Screening for Antimicrobial Activity}

The isolated colonies were subjected to cross streak method (for bacteria and yeasts) and dual inoculation method (for molds). In brief, the actinomycetes isolates were inoculated at the centre of sterile SCN agar plates as a single streak followed by incubation of plates aerobically at $30^{\circ} \mathrm{C}$ for $5-7$ days. Nutrient broth cultures of bacteria (Staphylococcus aureus, Bacillus cereus, $B$. subtilis, Streptococcus pyogenes, Escherichia coli, Pseudomonas aeruginosa, Shigella flexneri, Ralstonia solanacearum) and Sabouraud dextrose broth cultures of yeasts (Candida albicans, Cryptococcus neoformans) were inoculated by streaking perpendicular to the growth of actinomycetes but not touching the growth of actinomycetes. The molds (Aspergillus niger, A.flavus, Penicillium notatum, Fusarium oxysporum) were inoculated $1.5 \mathrm{~cm}$ away from the actinomycete growth by point inoculation. The plates were incubated at $37^{\circ} \mathrm{C}$ for 24 hours and $30^{\circ} \mathrm{C}$ for $48-72$ hours for bacteria and fungi respectively. Antimicrobial activity was determined by observing the distance of growth inhibition of test microorganisms from the growth of actinomycete isolates (Gautham, 2012). The SCN agar medium was supplemented with peptone (to facilitate the growth of test bacteria) and dextrose (to facilitate the growth of test fungi). Actinomycete isolates showing potent antagonistic activity were selected for further studies. 


\section{Characterization of Actinomycetes}

Actinomycetes isolates showing potent antimicrobial activity in primary screening were subjected to characterization by morphological (color of aerial and substrate mycelium, production of diffusible pigments), microscopic (cover slip method), staining (Gram's and Acid-fast staining) and biochemical tests (Starch hydrolysis, gelatin liquefaction, casein hydrolysis, cellulose hydrolysis, catalase test, oxidase test, nitrate reduction, H2S production, carbohydrate fermentation, citrase test) (Aneja, 1996; Cappuccino and Sherman, 1999; Gautham, 2012).

\section{Fermentation}

Potent actinomycetes isolates were cultivated in conical flasks containing sterile SCN broth medium. The isolates were inoculated into broth medium aseptically and incubated at $30^{\circ} \mathrm{C}$ for about 10 days. During incubation, the flasks were observed constantly for contamination, if any. After incubation, the contents of the flasks were filtered aseptically through sterile Muslin cloth followed by sterile Whatman filter paper No. 1 (Kekuda et al., 2013a).

\section{Solvent Extraction}

The crude culture filtrates were then subjected for solvent extraction in separation funnel using solvents viz., hexane, ethyl acetate and butanol. In separation funnel, culture filtrate and solvent were taken in 1:1 proportion and agitated well to ensure proper mixing of filtrate and solvent. The separation funnel was left for about 15 minutes in order to allow separation of organic phase (solvent) from aqueous phase (Alimuddin et al., 2011). Further, the solvent layer was separated, dried and the crude solvent extract was stored in sterile container.

\section{Antimicrobial Activity of Solvent Extracts}

Agar well diffusion method was performed to screen antimicrobial efficacy of solvent extracts of selected actinomycetes. In brief, the test bacteria ( 24 hours old nutrient broth cultures of $E$. coli, Klebsiella pneumoniae, Enterobacter aerogenes, Vibrio cholerae, P. aeruginosa, Xanthomonas campestris, $R$. solanacearum, Shigella dysenteriae, S. flexneri, S. aureus, B. subtilis, B. cereus and S. pyogenes) and fungi (48 hours old Sabouraud's dextrose broth cultures of yeasts viz., $C$. albicans and $C$. neoformans and spore suspension of molds viz., A. niger, $A$. flavus, $P$. notatum, Rhizopus stolonifer and $F$. oxysporum) were swabbed uniformly over the surface of sterile Nutrient agar plates and Sabouraud's dextrose agar plates respectively using sterile cotton swabs. With the help of sterile cork borer, wells of $6 \mathrm{~mm}$ were punched in the inoculated plates and the solvent extracts and standard antibiotic were added to respectively labeled wells. The plates were incubated at $37^{\circ} \mathrm{C}$ for 24 hours in case of bacteria and $48-72$ hours at $30^{\circ} \mathrm{C}$ in case of fungi. The zones of inhibition formed around the wells were measured (Kekuda et al., 2013a).

\section{Cytotoxic Activity of Solvent Extracts of Selected Actinomycetes}

Among three solvent extracts, the solvent extract showing marked antimicrobial activity was subjected to Brine shrimp lethality assay according to the method of McLaughlin (1991). The different concentrations of solvent extracts $(10-1000 \mu \mathrm{g} / \mathrm{ml})$ were prepared in methanol. Eggs of brine shrimp Artemia salina (Nihon Animal Pharmaceutical Inc., Tokyo, Japan) were hatched in a container filled with air-bubbled artificial sea water which was prepared using $10 \mathrm{~g}$ of a commercial salt mixture (GEX Inc., Osaka, Japan) and $500 \mathrm{ml}$ of distilled water. After $36-48 \mathrm{~h}$, the phototropic shrimps were collected by pipette and used for bioassay. The different concentrations of positive control (potassium dichromate) and solvent extracts were tested in vials containing $5 \mathrm{ml}$ of brine and 25 shrimps. Twenty five shrimps were transferred to each vial, artificial sea water was added to make up to $5 \mathrm{ml}$, incubated at $25^{\circ} \mathrm{C}$ and surviving shrimps were counted microscopically after 24 hours. Mortality of shrimps (\%) was calculated. LC $_{50}$ (lethal concentration) value was calculated using Origin 6 software. LC $_{50}$ refers to the concentration of extract required to cause $50 \%$ mortality of brine shrimp larvae.

\section{Antioxidant Activity of Solvent Extracts of Selected Actinomycetes}

Among three solvent extracts, the solvent extract showing promising antimicrobial activity was subjected to antioxidant activity by DPPH (1,1-diphenyl-2picrylhydrazyl), ABTS (2,2'-azino-bis-3-ethylbenzthia zoline-6-sulphonic acid), Metal chelating and Ferric reducing assay.

\section{DPPH Free Radical Scavenging Activity of Solvent Extracts}

In order to evaluate the radical scavenging activity of solvent extracts of actinomycete isolates, DPPH free radical scavenging assay was performed (Kekuda et al., 2010b). Solvent extracts and DPPH were prepared in methanol. Briefly, $2 \mathrm{ml}$ of DPPH solution $(0.002 \%$ in methanol) was mixed with $2 \mathrm{ml}$ of different concentrations $(0-200 \mu \mathrm{g} / \mathrm{ml})$ of solvent extracts and reference standard (ascorbic acid) in separate tubes. The tubes were incubated in dark at room temperature for 30 minutes and the optical density was measured at $517 \mathrm{~nm}$ using UVVisible spectrophotometer. The absorbance of the DPPH control (2ml methanol+2ml DPPH) was noted. The scavenging activity was calculated using the formula:

Scavenging activity $(\%)=[(A-B) / A] \times 100$,

where $A$ is absorbance of DPPH control and $B$ is absorbance of DPPH in the presence of extract/standard. $I_{50}$ (Inhibitory Concentration) value was calculated by Origin 6 software. The value denotes the concentration of extract/standard required to scavenge $50 \%$ of free radicals.

\section{ABTS Radical Scavenging Activity of Solvent Extracts}

The efficacy of solvent extracts to scavenge ABTS radicals was determined using the protocol of $\mathrm{Li}$ et al. (2011) with minor modifications. The ABTS radical was generated by reacting $7 \mathrm{mM}$ ABTS stock solution with $2.45 \mathrm{mM}$ potassium persulfate and the mixture was left at room temperature in the dark for 12-16 hours. The resulting solution was then diluted with distilled water to an absorbance of 0.70 at $730 \mathrm{~nm}$. $1 \mathrm{ml}$ of different concentrations $(0-200 \mu \mathrm{g} / \mathrm{ml})$ of solvent extracts was added to $4 \mathrm{ml}$ of ABTS radical solution and the tubes were incubated for 30 minutes followed by measuring the absorbance at $730 \mathrm{~nm}$. Ascorbic acid was used as reference standard. The radical-scavenging activity was calculated using the formula:

Scavenging activity $(\%)=[(A-B) / A] \times 100$, 
Prashith Kekuda et alo,

Where $A$ is the absorbance of the ABTS solution without extract/standard and $B$ is the absorbance of the test sample. $I_{50}$ value was calculated by Origin 6 software which denotes the concentration of extract/standard required to scavenge $50 \%$ of free radicals.

\section{Metal Chelating Activity of Solvent Extracts}

The chelating of ferrous ions by different concentrations of solvent extracts and EDTA (reference standard) was determined by following the protocol of Dinis et al. (1994). In brief, different concentrations (0$200 \mu \mathrm{g} / \mathrm{ml}$ ) of solvent extracts and standard were added to a solution of $\mathrm{FeCl}_{2}(2 \mathrm{mM}, 0.05 \mathrm{ml})$. The reaction was initiated by the addition of ferrozine $(5 \mathrm{mM}, 0.2 \mathrm{ml})$, the reaction mixture was shaken vigorously and left standing at room temperature for ten minutes. After the mixture reached equilibrium, the absorbance of the solution was measured at $562 \mathrm{~nm}$ in a spectrophotometer. The percentage of inhibition of ferrozine- $\mathrm{Fe}^{2+}$ complex formation was given by the formula:

Percent inhibition $=[(A-B) / A] \times 100$,

where $A$ was the absorbance of the control, and $B$ was the absorbance in the presence of the extract and standard. The control contains $\mathrm{FeCl}_{2}$ and ferrozine. $\mathrm{IC}_{50}$ value was calculated by Origin 6 software which denotes the concentration of extract/standard required to cause $50 \%$ chelation activity.

\section{Ferric Reducing Activity of Solvent Extracts}

The reducing effect of solvent extracts was tested by Ferric reducing assay. In this assay, different concentrations $(0-200 \mu \mathrm{g} / \mathrm{ml})$ of solvent extracts of isolates and ascorbic acid (reference standard) in $1 \mathrm{ml}$ of methanol were mixed separately with $2.5 \mathrm{ml}$ of phosphate buffer (200mM, pH 6.6) and $2.5 \mathrm{ml}$ of $1 \%$ potassium ferricyanide

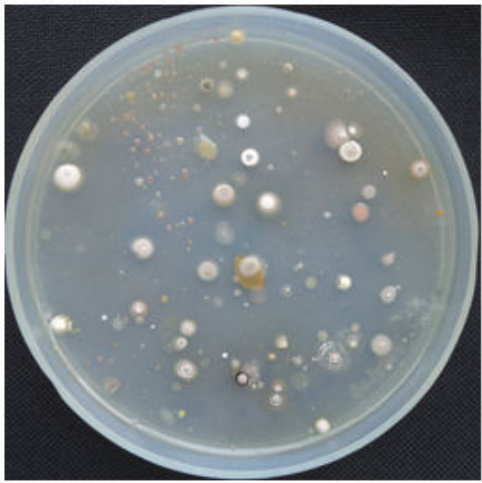

Figure 1: Actinomycetes colonies on SCN agar

\section{Primary Screening for Antimicrobial Activity}

All the isolates were subjected for primary screening to determine antimicrobial activity. On the basis of potent inhibition of test microbes, 17 isolates were selected for further studies and the isolates were designated as PO-01 to PO-16 and PO18. Table 2 shows the soil samples from which the potent actinomycetes isolates were recovered.

Table 3 shows the result of inhibitory effect of selected actinomycetes isolates against test bacteria in primary screening. All isolates were found inhibitory against one or more of the test bacteria. Three isolates viz., PO-05, PO10 and PO-13 caused inhibition of all test bacteria.
Sci. Technol. Arts Res. J., April-June 2015, 4(2): 164-180

followed by incubating reaction mixtures at $50^{\circ} \mathrm{C}$ for 20 minutes in water bath. The tubes were cooled rapidly and mixed with $2.5 \mathrm{ml}$ of $10 \%$ trichloroacetic acid and $0.5 \mathrm{ml}$ of $0.1 \%$ ferric chloride. After 10 minutes, the quantity of iron (II)-ferricyanide complex formed was determined by measuring the formation of Perl's Prussian blue at 700nm An increase in absorbance on increase in concentration denotes increased reducing power (Kekuda et al., 2010b).

\section{RESULTS}

\section{Isolation of Actinomycetes}

A total of 182 isolates were isolated on SCN agar from 57 soil samples collected at 13 areas of Western Ghats of Shivamogga district, Karnataka (Table 1). The isolates were identified as actinomycetes based on typical colony features. Figure 1 shows the dilution plates with colonies of actinomycetes.

Table 1: Number of actinomycetes isolated from soil samples

\begin{tabular}{|c|c|c|}
\hline Place & $\begin{array}{c}\text { Number of } \\
\text { soil samples }\end{array}$ & $\begin{array}{c}\text { Number of } \\
\text { actinomycetes }\end{array}$ \\
\hline Mandagadde & 6 & 13 (S01-S13) \\
\hline Maaluru & 4 & 11 (S14-S24) \\
\hline Tooduru & 3 & 9 (S25-S33) \\
\hline Mahishi & 4 & 14 (S34-S47) \\
\hline Bejjavalli & 4 & $13(S 48-60)$ \\
\hline Kudumallige & 3 & 11 (S61-71) \\
\hline Thirthahalli & 6 & 22 (S72-93) \\
\hline Kaimara & 4 & 11 (S94-104) \\
\hline Megaravalli & 6 & 15 (S105-119) \\
\hline Naaluru & 5 & 16 (S120-S135) \\
\hline Guddekeri & 4 & 11 (S136-S146) \\
\hline Kavarihaklu & 5 & 19 (S147-165) \\
\hline \multirow[t]{2}{*}{ Agumbe } & 3 & 18 (S166-S182) \\
\hline & Total $=57$ & Total $=182$ \\
\hline
\end{tabular}

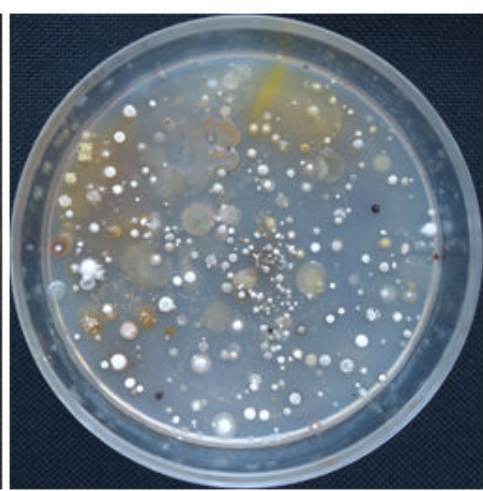

Overall, susceptibility of Gram positive bacteria was marked when compared to Gram negative bacteria. $S$. aureus and $P$. aeruginosa were inhibited to high extent among Gram positive and Gram negative bacteria respectively.

Table 4 shows the result of inhibitory potential of selected actinomycetes against test fungi in primary screening. Out of 17 isolates, 2 isolates (PO-05 and PO11 ) inhibited all test fungi. Marked inhibitory activity was observed in case of isolate PO-05. Overall, $C$. neoformans and $A$. flavus were inhibited to higher extent among yeasts and molds respectively. 
Table 2: Actinomycetes with potent antimicrobial activity and their origin (soil)

\begin{tabular}{cc}
\hline Isolates & Origin (soil) \\
\hline PO-01, PO-02 & Mandagadde \\
PO-03, PO-04 & Mahishi \\
PO-05 & Bejjavalli \\
PO-06 & Kudumallige \\
PO-07, PO-08 & Maaluru \\
PO-09, PO-10 & Thirthahalli \\
PO-11 & Kaimara \\
PO-12 & Megaravalli \\
PO-13 & Kavarihaklu \\
PO-14, PO-15 & Naaluru \\
PO-16, PO-18 & Agumbe \\
\hline
\end{tabular}

Table 3: Primary screening for antibacterial activity of selected actinomycetes

\begin{tabular}{|c|c|c|c|c|c|c|c|c|}
\hline \multirow{2}{*}{ Isolates } & \multicolumn{8}{|c|}{ Extent of inhibition of test bacteria } \\
\hline & $\mathrm{Sa}$ & $B c$ & Bs & Sp & $E c$ & $\mathrm{~Pa}$ & $S f$ & Rs \\
\hline PO-01 & ++ & + & + & + & - & + & - & + \\
\hline PO-02 & +++ & + & ++ & + & - & + & + & - \\
\hline PO-03 & + & + & + & + & - & + & - & - \\
\hline PO-04 & + & + & + & + & - & + & + & + \\
\hline PO-05 & +++ & ++ & + & ++ & + & ++ & + & + \\
\hline $\mathrm{PO}-06$ & ++ & ++ & ++ & + & - & + & + & + \\
\hline $\mathrm{PO}-07$ & + & + & + & + & - & ++ & + & ++ \\
\hline PO-08 & ++ & + & + & ++ & + & + & - & - \\
\hline PO-09 & ++ & + & + & + & - & ++ & - & - \\
\hline PO-10 & +++ & + & ++ & ++ & + & ++ & + & + \\
\hline PO-11 & ++ & + & + & - & + & + & + & + \\
\hline PO-12 & ++ & + & ++ & + & + & + & - & + \\
\hline PO-13 & +++ & ++ & +++ & ++ & + & ++ & + & + \\
\hline PO-14 & ++ & + & + & + & + & + & - & - \\
\hline PO-15 & + & + & + & + & - & + & - & - \\
\hline PO-16 & ++ & ++ & + & + & - & + & - & - \\
\hline PO-18 & ++ & + & ++ & ++ & - & + & - & + \\
\hline
\end{tabular}

Sa- S.aureus; Bc- B.cereus; Bs- B.subtilis; Sp-S.pyogenes; Ec- E.coli; Pa- P.aeruginosa; Sf- S.flexneri; Rs- R.solanacearum

Table 4: Primary screening for antifungal activity of selected actinomycetes

\begin{tabular}{ccccccc}
\hline \multirow{2}{*}{ Isolates } & \multicolumn{7}{c}{ Extent of inhibition of test fungi } \\
\cline { 2 - 7 } & Ca & Cn & An & Af & Pn & Fo \\
\hline $\mathrm{PO}-01$ & + & ++ & - & - & - & + \\
$\mathrm{PO}-02$ & + & ++ & + & + & - & + \\
$\mathrm{PO}-03$ & - & + & + & + & + & + \\
$\mathrm{PO}-04$ & + & ++ & + & + & - & + \\
$\mathrm{PO}-05$ & ++ & ++ & + & ++ & + & + \\
$\mathrm{PO}-06$ & - & - & + & - & - & + \\
$\mathrm{PO}-07$ & + & + & - & + & - & - \\
$\mathrm{PO}-08$ & - & + & - & + & - & - \\
$\mathrm{PO}-09$ & + & ++ & + & + & - & + \\
$\mathrm{PO}-10$ & + & + & + & + & - & + \\
$\mathrm{PO}-11$ & + & ++ & + & ++ & + & + \\
$\mathrm{PO}-12$ & + & ++ & - & + & - & + \\
$\mathrm{PO}-13$ & - & + & - & - & - & - \\
$\mathrm{PO}-14$ & - & ++ & + & + & - & + \\
$\mathrm{PO}-15$ & ++ & ++ & - & + & - & - \\
$\mathrm{PO}-16$ & + & ++ & + & + & - & + \\
$\mathrm{PO}-18$ & + & + & - & - & - & - \\
\hline
\end{tabular}

Ca- C.albicans; Cn- C.neoformans; An- A.niger; Af-A.flavus; Pn-P.notatum; Fo- F.oxysporum

\section{Characterization of Selected Actinomycetes}

Cultural, Staining and Microscopic Characteristics of Isolates

The cultural and microscopic characteristics of selected actinomycetes isolates are shown in Table 5 and Figure 2-6. The colonies exhibit characteristics such as raised, powdery, rugose, radiating, discrete and velvety. The colors of aerial mycelia were dark grey, grey, reddish grey, grayish brown, white, grayish white and yellow. The substrate mycelia were of different colors such as grey, light grey, brown, red and white. Spore arrangements of various kinds were observed in case of isolates. The spore characteristics observed were spiral, loop, straight, hook and flexibilis. Isolate PO-05 was found to produce diffusible pigment which is light brown in color. All isolates were Gram positive and non-acid fast.

\section{Biochemical Characteristics of Isolates}

Table 6 and Table 7 show the result of biochemical characteristics of isolates. All isolates were positive for starch hydrolysis and cellulose hydrolysis. All isolates exhibited proteolytic activity as indicated by liquefaction of gelatin or hydrolysis of casein or both. Catalse and nitrate reduction tests were positive in case of all isolates. Out of 17 isolates, 10 and 14 isolates were positive for gelatin liquefaction and casein hydrolysis respectively. Both gelatin liquefaction and casein hydrolysis were positive in case of 7 isolates. 15 out of 17 isolates were positive for citrase production. 13 isolates produced $\mathrm{H}_{2} \mathrm{~S}$ in SIM agar. The isolates were found to cause fermentation of one or more carbohydrates. Out of 17 isolates, 3 isolates fermented all carbohydrates. Only acid production (yellow color) was observed in case of glucose, fructose, galactose and mannitol. Alkali production (pink color) was observed in case of maltose and lactose. Gas production was not observed.

\section{Antibacterial Activity of Solvent Extracts}

Inhibitory effect of solvent extracts of selected actinomycetes against Gram positive and Gram negative bacteria is shown in Table 8 and Table 9. Ethyl acetate was found to be the best solvent for extraction of bioactive metabolites from 12 isolates viz., PO-01, PO-02, PO-03, $\mathrm{PO}-05$, PO-06, PO-08, PO-09, PO-10, PO-12, PO-15, $\mathrm{PO}-16$ and $\mathrm{PO}-18$ as the ethyl acetate extracts of these isolates caused marked inhibitory activity against test bacteria than other solvent extracts. Butanol extract of five isolates viz., PO-04, PO-07, PO-11, PO-13 and PO-14 exhibited stronger inhibitory activity than other solvent extracts. Least inhibitory effect was observed against test bacteria by hexane extracts. Hexane extract from most of the isolates did not inhibit any of the test bacteria. Overall, Gram positive bacteria were more susceptible to solvent extracts.

\section{Antifungal Activity of Solvent Extracts}

Table 10 and Table 11 depict inhibitory effect of solvent extracts of selected actinomycetes against test fungi. Butanol extract of 6 isolates (PO-04, PO-07, PO-11, $\mathrm{PO}-13, \mathrm{PO}-14$ and $\mathrm{PO}-18$ ) exhibited stronger antifungal activity than other solvent extracts. However, ethyl acetate extracts of 11 isolates caused higher inhibition of test fungi than other solvent extracts. Hexane extracts of isolates were least inhibitory against test fungi. Among yeasts, susceptibility was marked in case of $C$. neoformans when compared to $C$. albicans. 
Prashith Kekuda et al.,
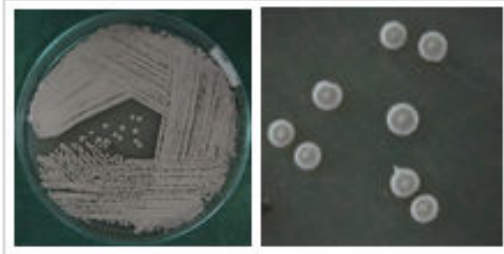

Isolate PO-01
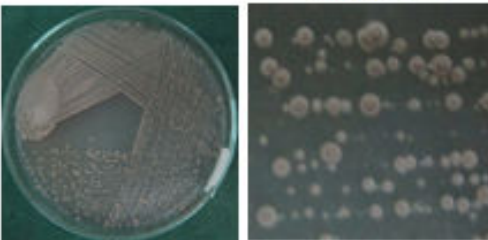

Isolate PO-02
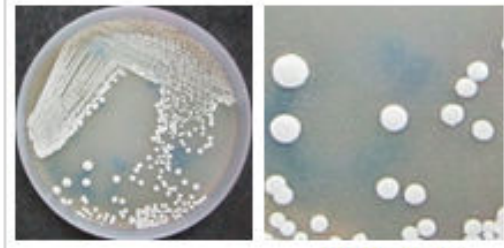

Isolate $\mathrm{PO}-03$
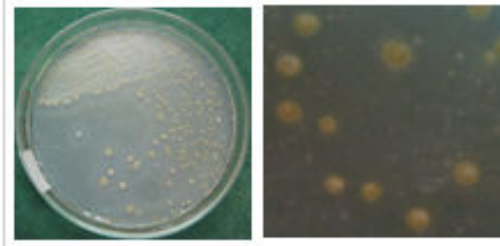

Isolate PO-04

Figure 2: Culture and spore arrangement of isolates (PO-01 to PO-04)
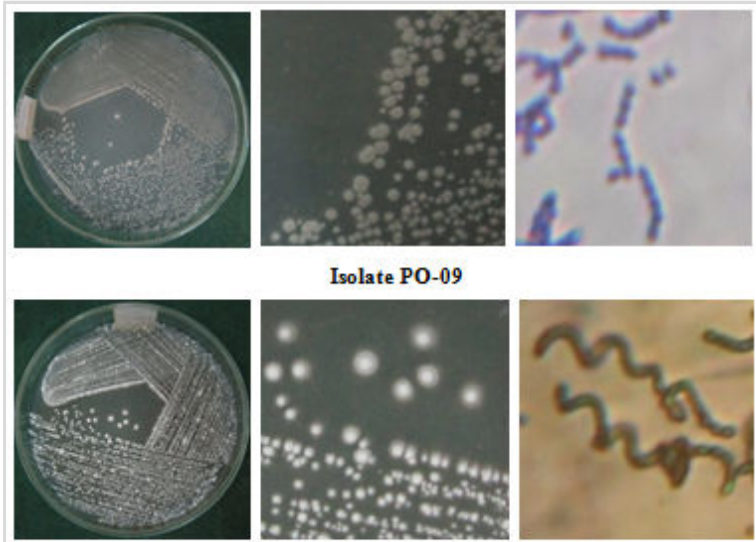

Isolate PO-10
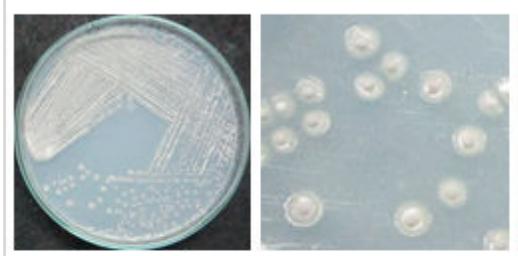

Isolate PO-11

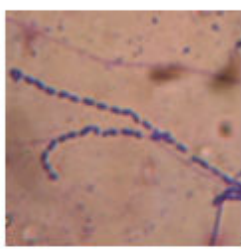

Figure 4: Culture and spore arrangement of isolates (PO-09 to PO-11)
Sci. Technol. Arts Res. J., April-June 2015, 4(2): 164-180
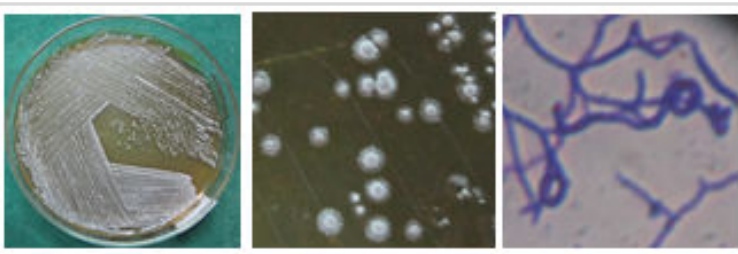

Isolate PO-05
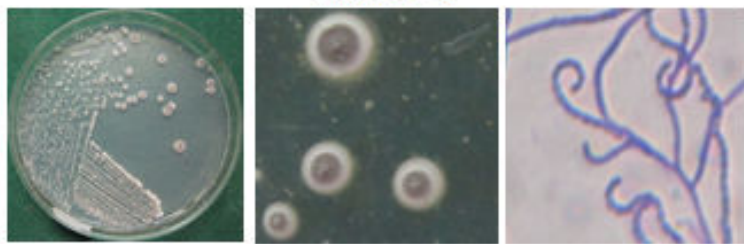

Isolate PO-06
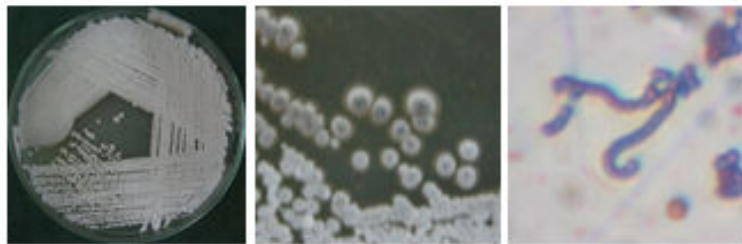

Isolate PO-07
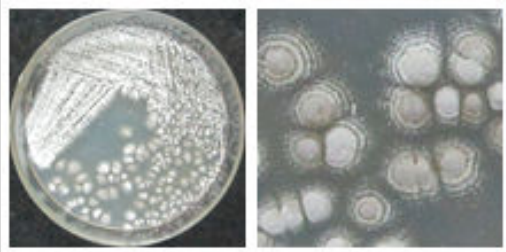

Isolate PO-08

Figure 3: Culture and spore arrangement of isolates (PO-05 to PO-08)
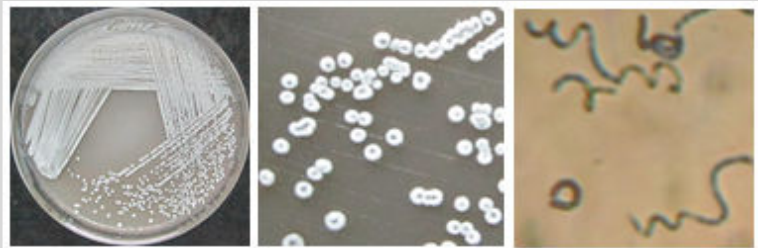

Isolate PO-12
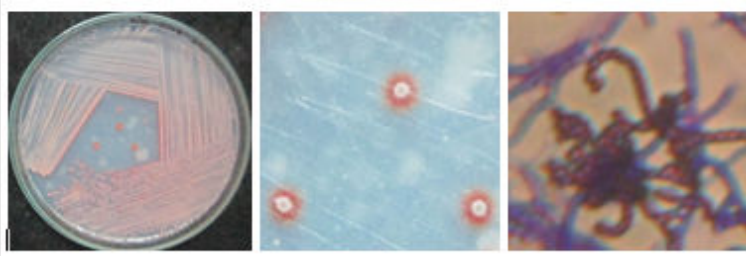

Isolate PO-13
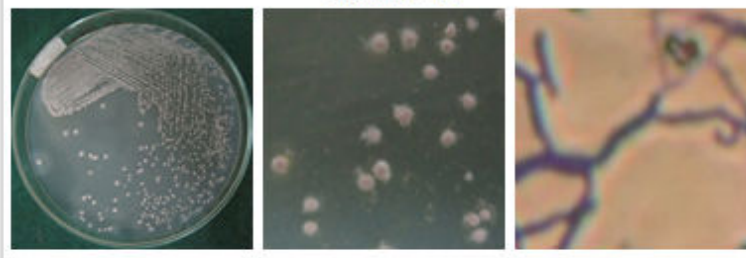

Isolate PO-14

Figure 5: Culture and spore arrangement of isolates (PO-12 to PO-14) 

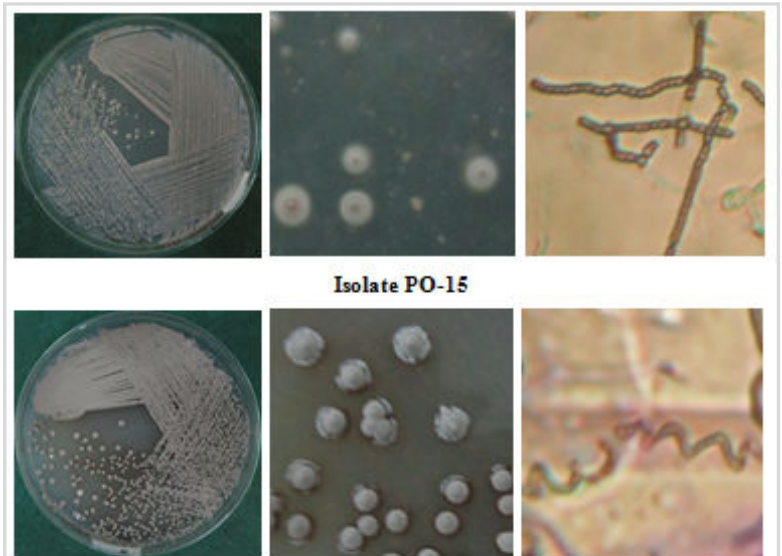

Isolate PO-15

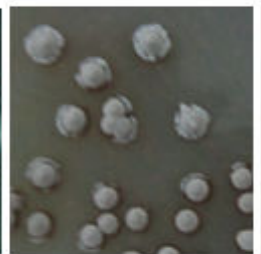

Isolate PO-16
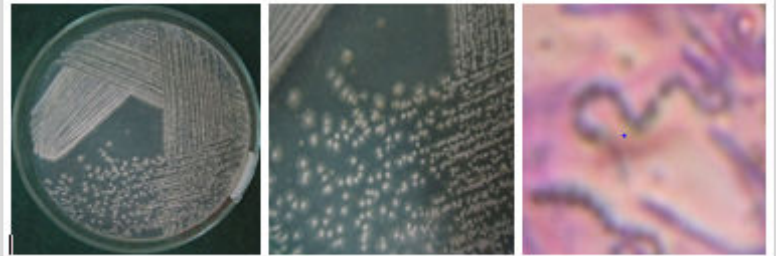

Isolate PO-18

Figure 6: Culture and spore arrangement of isolates (PO-15, PO-16 and PO-18)

Table 5: Cultural characteristics of selected actinomycetes

\begin{tabular}{|c|c|c|c|c|c|c|}
\hline Isolate & Colony & Aerial mycelium & $\begin{array}{l}\text { Substrate } \\
\text { mycelium }\end{array}$ & $\begin{array}{c}\text { Pigment } \\
\text { (Diffusible) }\end{array}$ & $\begin{array}{c}\text { Spore } \\
\text { arrangement }\end{array}$ & $\begin{array}{l}\text { Tentative } \\
\text { genera }\end{array}$ \\
\hline $\mathrm{PO}-01$ & Powdery & Dark grey & Brown & - & Loop & Streptomyces \\
\hline PO-02 & Powdery & Grayish brown & Brown & - & Hook & Streptomyces \\
\hline PO-03 & Raised, compact & White & White & - & Spiral & Streptomyces \\
\hline $\mathrm{PO}-04$ & Radiating & Yellow & White & - & Spiral & Streptomyces \\
\hline PO-05 & Discrete, radiating & Grey & Light grey & Light brown & Loop & Streptomyces \\
\hline PO-06 & Radiating, rugose & Grey & White & - & Hook & Streptomyces \\
\hline PO-07 & Rugose & Grey & White & - & Hook & Streptomyces \\
\hline PO-08 & Radiating & Grayish white & White & - & Flexibilis & Streptomyces \\
\hline PO-09 & Discrete, radiating & Grey & White & - & Straight & Streptomyces \\
\hline PO-10 & Radiating & White & White & - & Spiral & Streptomyces \\
\hline PO-11 & Radiating & Grey & White & - & Straight & Streptomyces \\
\hline $\mathrm{PO}-12$ & Discrete & Grey & Grey & - & Spiral & Streptomyces \\
\hline $\mathrm{PO}-13$ & Discrete & Reddish grey & Red & - & Hook & Streptomyces \\
\hline PO-14 & Discrete, radiating & Grey & Grey & - & Hook & Streptomyces \\
\hline PO-15 & Rugose & Grey & Grey & - & Straight & Streptomyces \\
\hline PO-16 & Powdery, radiating & Grey & Brown & - & Spiral & Streptomyces \\
\hline PO-18 & Discrete, radiating & Grey & Grey & - & Spiral & Streptomyces \\
\hline
\end{tabular}

Table 6: Biochemical characteristics of selected actinomycetes isolates

\begin{tabular}{|c|c|c|c|c|c|c|c|c|}
\hline Isolates & $\begin{array}{c}\text { Starch } \\
\text { hydrolysis }\end{array}$ & $\begin{array}{c}\text { Gelatin } \\
\text { liquefaction }\end{array}$ & $\begin{array}{c}\text { Casein } \\
\text { hydrolysis }\end{array}$ & $\begin{array}{c}\text { Cellulose } \\
\text { hydrolysis }\end{array}$ & $\begin{array}{c}\text { Catalase } \\
\text { test }\end{array}$ & $\begin{array}{c}\text { Nitrate } \\
\text { reduction }\end{array}$ & $\begin{array}{c}\text { Citrate } \\
\text { test }\end{array}$ & $\begin{array}{c}\mathrm{H}_{2} \mathrm{~S} \\
\text { production }\end{array}$ \\
\hline PO-01 & + & + & - & + & + & + & + & + \\
\hline PO-02 & + & - & + & + & + & + & + & + \\
\hline PO-03 & + & + & - & + & + & + & + & - \\
\hline PO-04 & + & - & + & + & + & + & + & + \\
\hline PO-05 & + & + & + & + & + & + & + & + \\
\hline PO-06 & + & - & + & + & + & + & - & + \\
\hline PO-07 & + & + & + & + & + & + & + & + \\
\hline PO-08 & + & + & + & + & + & + & + & + \\
\hline PO-09 & + & + & - & + & + & + & + & + \\
\hline PO-10 & + & + & + & + & + & + & - & - \\
\hline PO-11 & + & - & + & + & + & + & + & + \\
\hline PO-12 & + & - & + & + & + & + & + & + \\
\hline PO-13 & + & + & + & + & + & + & + & + \\
\hline PO-14 & + & - & + & + & + & + & + & - \\
\hline PO-15 & + & + & + & + & + & + & + & + \\
\hline PO-16 & + & + & + & + & + & + & + & + \\
\hline PO-18 & + & - & + & + & + & + & + & - \\
\hline
\end{tabular}


Table 7: Carbohydrate fermentation by selected actinomycetes isolates

\begin{tabular}{ccccccc}
\hline Isolates & Glucose & Fructose & Galactose & Maltose & Lactose & Mannitol \\
\hline $\mathrm{PO}-01$ & + & + & - & + & - & - \\
$\mathrm{PO}-02$ & + & - & + & - & + & + \\
$\mathrm{PO}-03$ & + & + & - & + & + & + \\
$\mathrm{PO}-04$ & - & - & + & + & - & - \\
$\mathrm{PO}-05$ & + & + & + & + & + & - \\
$\mathrm{PO}-06$ & + & - & + & - & + & + \\
$\mathrm{PO}-07$ & + & + & + & + & + & + \\
$\mathrm{PO}-08$ & + & + & + & + & + & + \\
$\mathrm{PO}-09$ & + & + & - & + & - & - \\
$\mathrm{PO}-10$ & + & + & + & + & + & + \\
$\mathrm{PO}-11$ & + & - & + & - & + & + \\
$\mathrm{PO}-12$ & + & - & + & + & + & + \\
$\mathrm{PO}-13$ & + & + & + & + & - & - \\
$\mathrm{PO}-14$ & + & - & + & + & + & + \\
$\mathrm{PO}-15$ & + & + & + & - & + & + \\
$\mathrm{PO}-16$ & + & + & + & + & + & - \\
$\mathrm{PO}-18$ & + & - & + & + & - & - \\
\hline
\end{tabular}

Table 8: Inhibitory activity of solvent extracts of selected actinomycetes (PO-01 to PO-09) against test bacteria

\begin{tabular}{|c|c|c|c|c|c|c|c|c|c|c|c|c|c|c|}
\hline \multirow{2}{*}{ I } & \multirow{2}{*}{ SE } & \multicolumn{13}{|c|}{ Test bacteria/Zone of inhibition in $\mathrm{cm}$ (Mean \pm SD) } \\
\hline & & $E c$ & $K p$ & $E a$ & $V c$ & $\mathrm{~Pa}$ & $X c$ & $R s$ & $S d$ & $S f$ & Sa & Bs & $B C$ & $S p$ \\
\hline \multirow{3}{*}{$\begin{array}{c}\mathrm{PO}- \\
01\end{array}$} & $\mathrm{H}$ & $0.0 \pm 0.0$ & $0.0 \pm 0.0$ & $0.0 \pm 0.0$ & $0.0 \pm 0.0$ & $0.0 \pm 0.0$ & $0.0 \pm 0.0$ & $0.0 \pm 0.0$ & $0.0 \pm 0.0$ & $0.0 \pm 0.0$ & $0.8 \pm 0.0$ & $0.0 \pm 0.0$ & $0.0 \pm 0.0$ & $0.0 \pm 0.0$ \\
\hline & EA & $0.8 \pm 0.0$ & $1.0 \pm 0.0$ & $0.8 \pm 0.0$ & $1.2 \pm 0.0$ & $1.2 \pm 0.1$ & $1.0 \pm 0.1$ & $0.8 \pm 0.0$ & $1.0 \pm 0.0$ & $0.0 \pm 0.0$ & $1.8 \pm 0.2$ & $1.4 \pm 0.1$ & $1.4 \pm 0.0$ & $1.6 \pm 0.2$ \\
\hline & B & $0.0 \pm 0.0$ & $0.8 \pm 0.0$ & $0.0 \pm 0.0$ & $0.0 \pm 0.0$ & $0.8 \pm 0.0$ & $0.0 \pm 0.0$ & $0.0 \pm 0.0$ & $0.0 \pm 0.0$ & $0.0 \pm 0.0$ & $0.8 \pm 0.0$ & $1.0 \pm 0.0$ & $1.2 \pm 0.0$ & $0.8 \pm 0.0$ \\
\hline \multirow{3}{*}{$\begin{array}{c}\mathrm{PO}- \\
02\end{array}$} & $\mathrm{H}$ & $0.0 \pm 0.0$ & $0.0 \pm 0.0$ & $0.0 \pm 0.0$ & $0.0 \pm 0.0$ & $0.0 \pm 0.0$ & $0.0 \pm 0.0$ & $0.0 \pm 0.0$ & $0.0 \pm 0.0$ & $0.0 \pm 0.0$ & $0.0 \pm 0.0$ & $0.0 \pm 0.0$ & $0.0 \pm 0.0$ & $0.0 \pm 0.0$ \\
\hline & EA & $0.0 \pm 0.0$ & $0.0 \pm 0.0$ & $0.0 \pm 0.0$ & $0.8 \pm 0.0$ & $1.2 \pm 0.0$ & $0.0 \pm 0.0$ & $1.3 \pm 0.0$ & $0.8 \pm 0.0$ & $0.0 \pm 0.0$ & $1.4 \pm 0.1$ & $1.3 \pm 0.0$ & $1.6 \pm 0.1$ & $1.8 \pm 0.1$ \\
\hline & B & $0.0 \pm 0.0$ & $0.0 \pm 0.0$ & $0.0 \pm 0.0$ & $0.0 \pm 0.0$ & $0.8 \pm 0.0$ & $0.0 \pm 0.0$ & $0.8 \pm 0.0$ & $0.0 \pm 0.0$ & $0.0 \pm 0.0$ & $0.0 \pm 0.0$ & $0.0 \pm 0.0$ & $0.8 \pm 0.0$ & $0.8 \pm 0.0$ \\
\hline \multirow{3}{*}{$\begin{array}{c}\mathrm{PO}- \\
03\end{array}$} & $\mathrm{H}$ & $0.0 \pm 0.0$ & $0.0 \pm 0.0$ & $0.0 \pm 0.0$ & $0.0 \pm 0.0$ & $0.0 \pm 0.0$ & $0.0 \pm 0.0$ & $0.0 \pm 0.0$ & $0.0 \pm 0.0$ & $0.0 \pm 0.0$ & $0.0 \pm 0.0$ & $0.0 \pm 0.0$ & $0.0 \pm 0.0$ & $0.0 \pm 0.0$ \\
\hline & EA & $0.8 \pm 0.0$ & $0.0 \pm 0.0$ & $0.8 \pm 0.0$ & $1.0 \pm 0.0$ & $1.0 \pm 0.0$ & $0.0 \pm 0.0$ & $0.8 \pm 0.0$ & $0.8 \pm 0.0$ & $0.0 \pm 0.0$ & $1.3 \pm 0.0$ & $1.1 \pm 0.0$ & $1.2 \pm 0.0$ & $0.8 \pm 0.0$ \\
\hline & B & $0.0 \pm 0.0$ & $0.0 \pm 0.0$ & $0.0 \pm 0.0$ & $0.0 \pm 0.0$ & $0.8 \pm 0.0$ & $0.0 \pm 0.0$ & $0.0 \pm 0.0$ & $0.0 \pm 0.0$ & $0.0 \pm 0.0$ & $0.8 \pm 0.0$ & $0.0 \pm 0.0$ & $0.0 \pm 0.0$ & $0.8 \pm 0.0$ \\
\hline \multirow{3}{*}{$\begin{array}{c}\text { PO- } \\
04\end{array}$} & $\mathrm{H}$ & $0.0 \pm 0.0$ & $0.8 \pm 0.0$ & $0.0 \pm 0.0$ & $0.0 \pm 0.0$ & $0.8 \pm 0.0$ & $0.0 \pm 0.0$ & $0.0 \pm 0.0$ & $0.0 \pm 0.0$ & $0.0 \pm 0.0$ & $0.8 \pm 0.0$ & $0.0 \pm 0.0$ & $0.0 \pm 0.0$ & $0.0 \pm 0.0$ \\
\hline & EA & $0.0 \pm 0.0$ & $0.8 \pm 0.0$ & $0.0 \pm 0.0$ & $0.0 \pm 0.0$ & $0.8 \pm 0.0$ & $0.0 \pm 0.0$ & $0.0 \pm 0.0$ & $0.0 \pm 0.0$ & $0.0 \pm 0.0$ & $0.8 \pm 0.0$ & $0.0 \pm 0.0$ & $0.0 \pm 0.0$ & $0.0 \pm 0.0$ \\
\hline & B & $0.0 \pm 0.0$ & $1.2 \pm 0.0$ & $0.0 \pm 0.0$ & $0.8 \pm 0.0$ & $1.2 \pm 0.0$ & $0.8 \pm 0.0$ & $1.0 \pm 0.1$ & $0.0 \pm 0.0$ & $0.8 \pm 0.0$ & $1.4 \pm 0.2$ & $1.2 \pm 0.0$ & $1.2 \pm 0.1$ & $1.2 \pm 0.0$ \\
\hline \multirow{3}{*}{$\begin{array}{c}\text { PO- } \\
05\end{array}$} & $\mathrm{H}$ & $0.0 \pm 0.0$ & $0.0 \pm 0.0$ & $0.0 \pm 0.0$ & $0.0 \pm 0.0$ & $0.0 \pm 0.0$ & $0.0 \pm 0.0$ & $0.0 \pm 0.0$ & $0.0 \pm 0.0$ & $0.0 \pm 0.0$ & $0.0 \pm 0.0$ & $0.0 \pm 0.0$ & $0.0 \pm 0.0$ & $0.0 \pm 0.0$ \\
\hline & EA & $0.0 \pm 0.0$ & $0.0 \pm 0.0$ & $0.0 \pm 0.0$ & $0.8 \pm 0.0$ & $0.0 \pm 0.0$ & $0.8 \pm 0.0$ & $0.0 \pm 0.0$ & $0.0 \pm 0.0$ & $0.0 \pm 0.0$ & $1.0 \pm 0.0$ & $1.2 \pm 0.0$ & $0.0 \pm 0.0$ & $0.8 \pm 0.0$ \\
\hline & B & $0.0 \pm 0.0$ & $0.0 \pm 0.0$ & $0.0 \pm 0.0$ & $0.0 \pm 0.0$ & $0.0 \pm 0.0$ & $0.0 \pm 0.0$ & $0.0 \pm 0.0$ & $0.0 \pm 0.0$ & $0.0 \pm 0.0$ & $0.8 \pm 0.0$ & $0.8 \pm 0.0$ & $0.0 \pm 0.0$ & $0.8 \pm 0.0$ \\
\hline \multirow{3}{*}{$\begin{array}{c}\text { PO- } \\
06\end{array}$} & $\mathrm{H}$ & $0.0 \pm 0.0$ & $0.0 \pm 0.0$ & $0.0 \pm 0.0$ & $0.0 \pm 0.0$ & $0.0 \pm 0.0$ & $0.0 \pm 0.0$ & $0.0 \pm 0.0$ & $0.0 \pm 0.0$ & $0.0 \pm 0.0$ & $0.8 \pm 0.0$ & $0.0 \pm 0.0$ & $0.0 \pm 0.0$ & $0.8 \pm 0.0$ \\
\hline & EA & $0.0 \pm 0.0$ & $0.0 \pm 0.0$ & $0.0 \pm 0.0$ & $0.0 \pm 0.0$ & $0.8 \pm 0.0$ & $0.0 \pm 0.0$ & $0.8 \pm 0.0$ & $0.0 \pm 0.0$ & $0.0 \pm 0.0$ & $0.8 \pm 0.0$ & $0.8 \pm 0.0$ & $0.0 \pm 0.0$ & $1.2 \pm 0.0$ \\
\hline & B & $0.0 \pm 0.0$ & $0.0 \pm 0.0$ & $0.0 \pm 0.0$ & $0.0 \pm 0.0$ & $0.0 \pm 0.0$ & $0.0 \pm 0.0$ & $0.0 \pm 0.0$ & $0.0 \pm 0.0$ & $0.0 \pm 0.0$ & $0.8 \pm 0.0$ & $0.0 \pm 0.0$ & $0.0 \pm 0.0$ & $0.8 \pm 0.0$ \\
\hline \multirow{3}{*}{$\begin{array}{c}\text { PO- } \\
07\end{array}$} & $\mathrm{H}$ & $0.0 \pm 0.0$ & $0.0 \pm 0.0$ & $0.0 \pm 0.0$ & $0.0 \pm 0.0$ & $0.0 \pm 0.0$ & $0.0 \pm 0.0$ & $0.0 \pm 0.0$ & $0.0 \pm 0.0$ & $0.0 \pm 0.0$ & $0.0 \pm 0.0$ & $0.0 \pm 0.0$ & $0.0 \pm 0.0$ & $0.0 \pm 0.0$ \\
\hline & EA & $0.0 \pm 0.0$ & $0.0 \pm 0.0$ & $0.0 \pm 0.0$ & $0.0 \pm 0.0$ & $0.0 \pm 0.0$ & $0.0 \pm 0.0$ & $0.0 \pm 0.0$ & $0.0 \pm 0.0$ & $0.0 \pm 0.0$ & $0.0 \pm 0.0$ & $0.0 \pm 0.0$ & $0.0 \pm 0.0$ & $0.0 \pm 0.0$ \\
\hline & B & $0.0 \pm 0.0$ & $0.0 \pm 0.0$ & $0.0 \pm 0.0$ & $0.0 \pm 0.0$ & $0.8 \pm 0.0$ & $0.0 \pm 0.0$ & $0.0 \pm 0.0$ & $0.0 \pm 0.0$ & $0.0 \pm 0.0$ & $0.8 \pm 0.0$ & $0.0 \pm 0.0$ & $1.2 \pm 0.0$ & $0.8 \pm 0.0$ \\
\hline \multirow{3}{*}{$\begin{array}{c}\mathrm{PO}- \\
08\end{array}$} & $\mathrm{H}$ & $0.0 \pm 0.0$ & $0.0 \pm 0.0$ & $0.0 \pm 0.0$ & $0.0 \pm 0.0$ & $0.0 \pm 0.0$ & $0.0 \pm 0.0$ & $0.0 \pm 0.0$ & $0.0 \pm 0.0$ & $0.0 \pm 0.0$ & $0.8 \pm 0.0$ & $0.0 \pm 0.0$ & $0.0 \pm 0.0$ & $0.8 \pm 0.0$ \\
\hline & EA & $0.8 \pm 0.0$ & $1.2 \pm 0.0$ & $0.8 \pm 0.0$ & $0.8 \pm 0.0$ & $1.4 \pm 0.0$ & $0.8 \pm 0.0$ & $1.2 \pm 0.0$ & $0.8 \pm 0.0$ & $0.0 \pm 0.0$ & $1.4 \pm 0.0$ & $1.2 \pm 0.0$ & $1.6 \pm 0.0$ & $1.4 \pm 0.0$ \\
\hline & B & $0.0 \pm 0.0$ & $0.0 \pm 0.0$ & $0.0 \pm 0.0$ & $0.0 \pm 0.0$ & $0.8 \pm 0.0$ & $0.0 \pm 0.0$ & $0.8 \pm 0.0$ & $0.0 \pm 0.0$ & $0.0 \pm 0.0$ & $1.0 \pm 0.0$ & $0.8 \pm 0.0$ & $0.8 \pm 0.0$ & $0.8 \pm 0.0$ \\
\hline \multirow{3}{*}{$\begin{array}{c}\text { PO- } \\
09\end{array}$} & $\mathrm{H}$ & $0.0 \pm 0.0$ & $0.0 \pm 0.0$ & $0.0 \pm 0.0$ & $0.0 \pm 0.0$ & $0.0 \pm 0.0$ & $0.0 \pm 0.0$ & $0.0 \pm 0.0$ & $0.0 \pm 0.0$ & $0.0 \pm 0.0$ & $0.0 \pm 0.0$ & $0.0 \pm 0.0$ & $0.0 \pm 0.0$ & $0.0 \pm 0.0$ \\
\hline & EA & $0.0 \pm 0.0$ & $0.0 \pm 0.0$ & $0.0 \pm 0.0$ & $0.8 \pm 0.0$ & $1.2 \pm 0.1$ & $0.0 \pm 0.0$ & $0.0 \pm 0.0$ & $0.0 \pm 0.0$ & $0.0 \pm 0.0$ & $1.6 \pm 0.2$ & $0.8 \pm 0.0$ & $1.0 \pm 0.0$ & $0.0 \pm 0.0$ \\
\hline & B & $0.0 \pm 0.0$ & $0.0 \pm 0.0$ & $0.0 \pm 0.0$ & $0.0 \pm 0.0$ & $0.8 \pm 0.0$ & $0.0 \pm 0.0$ & $0.0 \pm 0.0$ & $0.0 \pm 0.0$ & $0.0 \pm 0.0$ & $0.8 \pm 0.0$ & $0.0 \pm 0.0$ & $0.8 \pm 0.0$ & $0.0 \pm 0.0$ \\
\hline
\end{tabular}


Table 9: Inhibitory activity of solvent extracts of selected actinomycetes (PO-10 to PO-16 and PO-18) against test bacteria

\begin{tabular}{|c|c|c|c|c|c|c|c|c|c|c|c|c|c|c|}
\hline \multirow{2}{*}{ I } & \multirow{2}{*}{ E } & \multicolumn{13}{|c|}{ Test bacteria/Zone of inhibition in $\mathrm{cm}$ (Mean \pm SD) } \\
\hline & & $E c$ & Kp & Ea & $V c$ & $\mathrm{~Pa}$ & $X c$ & Rs & Sd & Sf & Sa & Bs & $B c$ & $S p$ \\
\hline \multirow{3}{*}{$\begin{array}{l}\text { PO- } \\
10\end{array}$} & H & $0.0 \pm 0.0$ & $.0 \pm 0.0$ & $.0 \pm 0.0$ & $0.0 \pm 0.0$ & $.0 \pm 0.0$ & $0.0 \pm 0.0$ & $0.0 \pm 0.0$ & $0.0 \pm 0.0$ & $0.0 \pm 0.0$ & $0.0 \pm 0.0$ & $0.0 \pm 0.0$ & $0.0 \pm 0.0$ & $0.0 \pm 0.0$ \\
\hline & EA & $0.8 \pm 0.0$ & $0.0 \pm 0.0$ & $0.8 \pm 0.0$ & $1.0 \pm 0.0$ & $1.2 \pm 0.2$ & $0.0 \pm 0.0$ & $0.8 \pm 0.0$ & $0.0 \pm 0.0$ & $0.0 \pm 0.0$ & $1.2 \pm 0.0$ & $1.0 \pm 0.0$ & $1.4 \pm 0.0$ & $1.2 \pm 0.0$ \\
\hline & B & $0.0 \pm 0.0$ & $.0 \pm 0.0$ & $0 \pm 0.0$ & $0.8 \pm 0.0$ & $.8 \pm 0.0$ & $0.0 \pm 0.0$ & $.0 \pm 0.0$ & $0.0 \pm 0.0$ & $.0 \pm 0.0$ & $0.8 \pm 0.0$ & $0.0 \pm 0.0$ & $0 \pm 0.0$ & $.8 \pm 0.0$ \\
\hline \multirow{3}{*}{$\begin{array}{l}\text { PO- } \\
11\end{array}$} & $\Pi$ & \pm 0.0 & $0 \pm 0.0$ & $0.0 \pm 0.0$ & $0.0 \pm 0.0$ & $0.0 \pm 0.0$ & 0.0 & $.0 \pm 0.0$ & $0.0 \pm 0.0$ & $.0 \pm 0.0$ & $0.0 \pm 0.0$ & $0.0 \pm 0.0$ & $0.0 \pm 0.0$ & $0.0 \pm 0.0$ \\
\hline & EA & $0.0 \pm 0.0$ & $0.0 \pm 0.0$ & $0.0 \pm 0.0$ & $0.0 \pm 0.0$ & $0.8 \pm 0.0$ & $0.0 \pm 0.0$ & $0.0 \pm 0.0$ & $0.0 \pm 0.0$ & $0.0 \pm 0.0$ & $0.8 \pm 0.0$ & $0.0 \pm 0.0$ & $0.8 \pm 0.0$ & $0.0 \pm 0.0$ \\
\hline & $B$ & $0.0 \pm 0.0$ & $0.0 \pm 0.0$ & $0.0 \pm 0.0$ & $0.8 \pm 0.0$ & $0.8 \pm 0.0$ & $0.0 \pm 0.0$ & $0.0 \pm 0.0$ & $0.0 \pm 0.0$ & $0.0 \pm 0.0$ & $1.0 \pm 0.1$ & $0.8 \pm 0.0$ & $1.2 \pm 0.2$ & $0.8 \pm 0.0$ \\
\hline \multirow{3}{*}{$\begin{array}{l}\mathrm{PO}- \\
12\end{array}$} & $\mathrm{H}$ & $.0 \pm 0.0$ & $0.0 \pm 0.0$ & $0.0 \pm 0.0$ & $0.0 \pm 0.0$ & $0.0 \pm 0.0$ & $0.0 \pm 0.0$ & $0.0 \pm 0.0$ & $0.0 \pm 0.0$ & $0.0 \pm 0.0$ & $0.0 \pm 0.0$ & $0.0 \pm 0.0$ & $0.0 \pm 0.0$ & $0.0 \pm 0.0$ \\
\hline & EA & $0.8 \pm 0.0$ & $1.0 \pm 0.0$ & $0.0 \pm 0.0$ & $1.2 \pm 0.0$ & $1.4 \pm 0.0$ & $0.0 \pm 0.0$ & $0.8 \pm 0.0$ & $0.0 \pm 0.0$ & $0.8 \pm 0.0$ & $1.6 \pm 0.2$ & $1.4 \pm 0.1$ & $1.8 \pm 0.2$ & $1.2 \pm 0.2$ \\
\hline & B & $0.0 \pm 0.0$ & $0.8 \pm 0.0$ & $0.0 \pm 0.0$ & $0.0 \pm 0.0$ & $0.8 \pm 0.0$ & $0.0 \pm 0.0$ & $0.8 \pm 0.0$ & $0.0 \pm 0.0$ & $0.0 \pm 0.0$ & $0.8 \pm 0.0$ & $0.8 \pm 0.0$ & $1.2 \pm 0.1$ & $0.8 \pm 0.0$ \\
\hline \multirow{3}{*}{$\begin{array}{l}\text { PO- } \\
13\end{array}$} & $\mathrm{H}$ & $0.0 \pm 0.0$ & $0.0 \pm 0.0$ & $0.0 \pm 0.0$ & $0.0 \pm 0.0$ & $0.0 \pm 0.0$ & $0.0 \pm 0.0$ & $0.0 \pm 0.0$ & $0.0 \pm 0.0$ & $0.0 \pm 0.0$ & $0.0 \pm 0.0$ & $0.8 \pm 0.0$ & $0.8 \pm 0.0$ & $0.0 \pm 0.0$ \\
\hline & EA & $0.0 \pm 0.0$ & $0.8 \pm 0.0$ & $0.0 \pm 0.0$ & $0.0 \pm 0.0$ & $0.8 \pm 0.0$ & $0.0 \pm 0.0$ & $0.0 \pm 0.0$ & $0.0 \pm 0.0$ & $0.0 \pm 0.0$ & $1.2 \pm 0.0$ & $1.4 \pm 0.0$ & $0.8 \pm 0.0$ & $0.8 \pm 0.0$ \\
\hline & B & $0.0 \pm 0.0$ & $0.8 \pm 0.0$ & $0.0 \pm 0.0$ & $0.8 \pm 0.0$ & $1.2 \pm 0.0$ & $0.0 \pm 0.0$ & $0.8 \pm 0.0$ & $0.0 \pm 0.0$ & $0.0 \pm 0.0$ & $1.8 \pm 0.2$ & $2.0 \pm 0.1$ & $1.6 \pm 0.2$ & $1.2 \pm 0.0$ \\
\hline \multirow{3}{*}{$\begin{array}{l}\text { PO- } \\
14\end{array}$} & $\mathrm{H}$ & $0.0 \pm 0.0$ & $0.0 \pm 0.0$ & $0.0 \pm 0.0$ & $0.0 \pm 0.0$ & $0.0 \pm 0.0$ & $0.0 \pm 0.0$ & $0.0 \pm 0.0$ & $0.0 \pm 0.0$ & $0.0 \pm 0.0$ & $0.0 \pm 0.0$ & $0.0 \pm 0.0$ & $0.0 \pm 0.0$ & $0.0 \pm 0.0$ \\
\hline & EA & $0.0 \pm 0.0$ & $0.0 \pm 0.0$ & $0.0 \pm 0.0$ & $0.0 \pm 0.0$ & $0.0 \pm 0.0$ & $0.0 \pm 0.0$ & $0.0 \pm 0.0$ & $0.0 \pm 0.0$ & $0.0 \pm 0.0$ & $0.8 \pm 0.0$ & $0.0 \pm 0.0$ & $0.8 \pm 0.0$ & $0.0 \pm 0.0$ \\
\hline & B & $0.0 \pm 0.0$ & $0.0 \pm 0.0$ & $0.0 \pm 0.0$ & $0.0 \pm 0.0$ & $0.8 \pm 0.0$ & $0.0 \pm 0.0$ & $0.0 \pm 0.0$ & $0.0 \pm 0.0$ & $0.0 \pm 0.0$ & $0.8 \pm 0.0$ & $0.0 \pm 0.0$ & $1.2 \pm 0.1$ & $0.8 \pm 0.0$ \\
\hline \multirow{3}{*}{$\begin{array}{l}\mathrm{PO}- \\
15\end{array}$} & $\mathrm{H}$ & $0.0 \pm 0.0$ & $0.0 \pm 0.0$ & $0.0 \pm 0.0$ & $0.0 \pm 0.0$ & $0.0 \pm 0.0$ & $0.0 \pm 0.0$ & $0.0 \pm 0.0$ & $0.0 \pm 0.0$ & $0.0 \pm 0.0$ & $0.0 \pm 0.0$ & $0.0 \pm 0.0$ & $0.0 \pm 0.0$ & $0.0 \pm 0.0$ \\
\hline & EA & .1 & 0.0 & 0.0 & 0.0 & 2 & $0.8 \pm$ & 0.8 & 1. & $0.0 \pm 0.0$ & .0 & \pm 0.0 & $=0.0$ & $1.6 \pm 0.0$ \\
\hline & B & $0.0 \pm 0.0$ & $0.0 \pm 0.0$ & $0.0 \pm 0.0$ & $0.0 \pm 0.0$ & $0.8 \pm 0.0$ & $0.0 \pm 0.0$ & $0.0 \pm 0.0$ & $0.0 \pm 0.0$ & $0.0 \pm 0.0$ & $0.8 \pm 0.0$ & $0.0 \pm 0.0$ & $0.0 \pm 0.0$ & $0.8 \pm 0.0$ \\
\hline \multirow{3}{*}{$\begin{array}{l}\text { PO- } \\
16\end{array}$} & $\mathrm{H}$ & $0.0 \pm 0.0$ & $0.0 \pm 0.0$ & $0.0 \pm 0.0$ & $0.0 \pm 0.0$ & $0.0 \pm 0.0$ & $0.0 \pm 0.0$ & $0.0 \pm 0.0$ & $0.0 \pm 0.0$ & $0.0 \pm 0.0$ & $0.0 \pm 0.0$ & $0.0 \pm 0.0$ & $0.0 \pm 0.0$ & $0.0 \pm 0.0$ \\
\hline & EA & $0.8 \pm 0.0$ & $0.0 \pm 0.0$ & $0.0 \pm 0.0$ & $0.0 \pm 0.0$ & $0.8 \pm 0.0$ & $0.0 \pm 0.0$ & $0.0 \pm 0.0$ & $0.0 \pm 0.0$ & $0.0 \pm 0.0$ & $1.2 \pm 0.1$ & $1.0 \pm 0.0$ & $0.8 \pm 0.0$ & $0.8 \pm 0.0$ \\
\hline & B & $0.0 \pm 0.0$ & $0.0 \pm 0.0$ & $0.0 \pm 0.0$ & $0.8 \pm 0.0$ & $0.0 \pm 0.0$ & $0.0 \pm 0.0$ & $0.0 \pm 0.0$ & $0.0 \pm 0.0$ & $0.0 \pm 0.0$ & $0.0 \pm 0.0$ & $0.8 \pm 0.0$ & $0.0 \pm 0.0$ & $0.0 \pm 0.0$ \\
\hline \multirow{3}{*}{$\begin{array}{l}\text { PO- } \\
18\end{array}$} & $\mathrm{H}$ & $0.0 \pm 0.0$ & $0.0 \pm 0.0$ & $0.0 \pm 0.0$ & $0.0 \pm 0.0$ & $0.0 \pm 0.0$ & $0.0 \pm 0.0$ & $0.0 \pm 0.0$ & $0.0 \pm 0.0$ & $0.0 \pm 0.0$ & $0.0 \pm 0.0$ & $0.0 \pm 0.0$ & $0.0 \pm 0.0$ & $0.0 \pm 0.0$ \\
\hline & EA & $0.8 \pm 0.0$ & $0.8 \pm 0.0$ & $0.0 \pm 0.0$ & $1.0 \pm 0.0$ & $1.2 \pm 0.0$ & $0.8 \pm 0.0$ & $0.0 \pm 0.0$ & $0.8 \pm 0.0$ & $0.0 \pm 0.0$ & $1.2 \pm 0.0$ & $1.4 \pm 0.1$ & $1.2 \pm 0.0$ & $1.6 \pm 0.2$ \\
\hline & B & $0.0 \pm 0.0$ & $0.0 \pm 0.0$ & $0.0 \pm 0.0$ & $0.0 \pm 0.0$ & $0.8 \pm 0.0$ & $0.0 \pm 0.0$ & $0.0 \pm 0.0$ & $0.0 \pm 0.0$ & $0.0 \pm 0.0$ & $0.8 \pm 0.0$ & $0.0 \pm 0.0$ & $0.0 \pm 0.0$ & $1.0 \pm 0.1$ \\
\hline
\end{tabular}

I- Isolate; SE- Solvent Extract; H- Hexane; EA- Ethyl acetate; B- Butanol; Ec- E. coli; Kp- K. pneumoniae; Ea- E. aerogenes; Vc- V. cholerae; Pa- P. aeruginosa; Xc- X. campestris; Rs- R. solanacearum; Sd- S. dysenteriae; Sf- S. flexneri; Sa- S. aureus; Bs- B. subtilis; Bc- B. cereus; Sp- S. pyogenes

Table 10: Inhibitory activity of solvent extracts of selected actinomycetes (PO-01 to PO-09) against test fungi

\begin{tabular}{|c|c|c|c|c|c|c|c|c|}
\hline \multirow{2}{*}{ Isolate } & \multirow{2}{*}{$\begin{array}{l}\text { Solvent } \\
\text { extract }\end{array}$} & \multicolumn{7}{|c|}{ Test fungi/Zone of inhibition in $\mathrm{cm}$ (Mean \pm SD) } \\
\hline & & C. albicans & C. neoformans & A. niger & A. flavus & P. notatum & R. stolonifer & F. oxysporum \\
\hline & $\mathrm{H}$ & $0.0 \pm 0.0$ & $0.0 \pm 0.0$ & $0.8 \pm 0.0$ & $0.0 \pm 0.0$ & $0.0 \pm 0.0$ & $0.0 \pm 0.0$ & $0.0 \pm 0.0$ \\
\hline \multirow[t]{3}{*}{ PO-01 } & EA & $0.8 \pm 0.0$ & $0.0 \pm 0.0$ & $0.8 \pm 0.0$ & $0.0 \pm 0.0$ & $0.8 \pm 0.0$ & $0.0 \pm 0.0$ & $1.0 \pm 0.0$ \\
\hline & $\mathrm{B}$ & $0.8 \pm 0.0$ & $0.0 \pm 0.0$ & $0.0 \pm 0.0$ & $0.0 \pm 0.0$ & $0.0 \pm 0.0$ & $0.0 \pm 0.0$ & $0.0 \pm 0.0$ \\
\hline & $\mathrm{H}$ & $0.0 \pm 0.0$ & $0.0 \pm 0.0$ & $0.8 \pm 0.0$ & $0.0 \pm 0.0$ & $0.0 \pm 0.0$ & $0.0 \pm 0.0$ & $0.0 \pm 0.0$ \\
\hline \multirow[t]{3}{*}{ PO-02 } & EA & $0.8 \pm 0.0$ & $1.2 \pm 0.1$ & $1.0 \pm 0.1$ & $0.0 \pm 0.0$ & $0.8 \pm 0.0$ & $0.0 \pm 0.0$ & $1.0 \pm 0.0$ \\
\hline & $\mathrm{B}$ & $0.0 \pm 0.0$ & $0.8 \pm 0.0$ & $0.8 \pm 0.0$ & $0.8 \pm 0.0$ & $0.0 \pm 0.0$ & $0.0 \pm 0.0$ & $0.8 \pm 0.0$ \\
\hline & $\mathrm{H}$ & $0.0 \pm 0.0$ & $0.0 \pm 0.0$ & $0.8 \pm 0.0$ & $0.0 \pm 0.0$ & $0.0 \pm 0.0$ & $0.0 \pm 0.0$ & $0.0 \pm 0.0$ \\
\hline \multirow[t]{3}{*}{ PO-03 } & EA & $1.0 \pm 0.1$ & $1.1 \pm 0.1$ & $0.8 \pm 0.0$ & $0.8 \pm 0.0$ & $1.0 \pm 0.0$ & $0.9 \pm 0.1$ & $0.8 \pm 0.0$ \\
\hline & $\mathrm{B}$ & $0.0 \pm 0.0$ & $0.8 \pm 0.0$ & $0.8 \pm 0.0$ & $0.0 \pm 0.0$ & $0.0 \pm 0.0$ & $0.0 \pm 0.0$ & $0.8 \pm 0.0$ \\
\hline & $\mathrm{H}$ & $0.0 \pm 0.0$ & $0.0 \pm 0.0$ & $0.0 \pm 0.0$ & $0.0 \pm 0.0$ & $0.0 \pm 0.0$ & $0.0 \pm 0.0$ & $0.0 \pm 0.0$ \\
\hline \multirow[t]{3}{*}{ PO-04 } & EA & $0.8 \pm 0.0$ & $0.9 \pm 0.1$ & $0.8 \pm 0.0$ & $1.2 \pm 0.0$ & $0.8 \pm 0.0$ & $0.0 \pm 0.0$ & $0.8 \pm 0.0$ \\
\hline & $\mathrm{B}$ & $0.8 \pm 0.0$ & $1.2 \pm 0.2$ & $1.1 \pm 0.1$ & $1.4 \pm 0.1$ & $1.0 \pm 0.0$ & $0.8 \pm 0.0$ & $1.2 \pm 0.2$ \\
\hline & $\mathrm{H}$ & $0.0 \pm 0.0$ & $0.0 \pm 0.0$ & $0.8 \pm 0.0$ & $0.0 \pm 0.0$ & $0.0 \pm 0.0$ & $0.0 \pm 0.0$ & $0.0 \pm 0.0$ \\
\hline \multirow[t]{3}{*}{ PO-05 } & EA & $1.1 \pm 0.2$ & $1.4 \pm 0.2$ & $1.2 \pm 0.2$ & $1.1 \pm 0.0$ & $1.0 \pm 0.0$ & $0.8 \pm 0.0$ & $0.8 \pm 0.1$ \\
\hline & B & $0.8 \pm 0.0$ & $0.8 \pm 0.0$ & $0.8 \pm 0.0$ & $0.0 \pm 0.0$ & $0.8 \pm 0.0$ & $0.0 \pm 0.0$ & $0.0 \pm 0.0$ \\
\hline & $\mathrm{H}$ & $0.0 \pm 0.0$ & $0.0 \pm 0.0$ & $0.0 \pm 0.0$ & $0.0 \pm 0.0$ & $0.0 \pm 0.0$ & $0.0 \pm 0.0$ & $0.0 \pm 0.0$ \\
\hline \multirow[t]{3}{*}{ PO-06 } & EA & $1.0 \pm 0.0$ & $1.2 \pm 0.0$ & $0.8 \pm 0.0$ & $1.0 \pm 0.0$ & $1.0 \pm 0.0$ & $0.0 \pm 0.0$ & $1.2 \pm 0.0$ \\
\hline & $\mathrm{B}$ & $0.8 \pm 0.0$ & $0.8 \pm 0.0$ & $0.8 \pm 0.0$ & $0.8 \pm 0.0$ & $0.8 \pm 0.0$ & $0.0 \pm 0.0$ & $0.8 \pm 0.0$ \\
\hline & $\mathrm{H}$ & $0.0 \pm 0.0$ & $0.0 \pm 0.0$ & $0.0 \pm 0.0$ & $0.0 \pm 0.0$ & $0.0 \pm 0.0$ & $0.0 \pm 0.0$ & $0.0 \pm 0.0$ \\
\hline \multirow[t]{3}{*}{ PO-07 } & EA & $0.8 \pm 0.0$ & $0.8 \pm 0.0$ & $0.8 \pm 0.0$ & $0.0 \pm 0.0$ & $0.8 \pm 0.0$ & $0.0 \pm 0.0$ & $0.0 \pm 0.0$ \\
\hline & B & $1.0 \pm 0.0$ & $1.2 \pm 0.1$ & $0.8 \pm 0.0$ & $0.8 \pm 0.0$ & $0.8 \pm 0.0$ & $0.0 \pm 0.0$ & $1.0 \pm 0.0$ \\
\hline & $\mathrm{H}$ & $0.0 \pm 0.0$ & $0.0 \pm 0.0$ & $0.8 \pm 0.0$ & $0.0 \pm 0.0$ & $0.0 \pm 0.0$ & $0.0 \pm 0.0$ & $0.0 \pm 0.0$ \\
\hline \multirow[t]{3}{*}{ PO-08 } & EA & $1.0 \pm 0.0$ & $1.2 \pm 0.0$ & $1.0 \pm 0.0$ & $1.0 \pm 0.0$ & $0.8 \pm 0.0$ & $0.0 \pm 0.0$ & $1.0 \pm 0.0$ \\
\hline & $\mathrm{B}$ & $0.8 \pm 0.0$ & $1.0 \pm 0.0$ & $0.8 \pm 0.0$ & $0.8 \pm 0.0$ & $0.0 \pm 0.0$ & $0.0 \pm 0.0$ & $0.0 \pm 0.0$ \\
\hline & $\mathrm{H}$ & $0.0 \pm 0.0$ & $0.8 \pm 0.0$ & $0.8 \pm 0.0$ & $0.0 \pm 0.0$ & $0.0 \pm 0.0$ & $0.0 \pm 0.0$ & $0.0 \pm 0.0$ \\
\hline \multirow[t]{2}{*}{ PO-09 } & EA & $1.3 \pm 0.2$ & $1.3 \pm 0.1$ & $1.0 \pm 0.0$ & $0.8 \pm 0.0$ & $0.8 \pm 0.0$ & $0.8 \pm 0.0$ & $1.2 \pm 0.2$ \\
\hline & $\mathrm{B}$ & $0.0 \pm 0.0$ & $0.0 \pm 0.0$ & $0.8 \pm 0.0$ & $0.0 \pm 0.0$ & $0.0 \pm 0.0$ & $0.0 \pm 0.0$ & $0.0 \pm 0.0$ \\
\hline
\end{tabular}


Prashith Kekuda et alo,

Sci. Technol. Arts Res. J., April-June 2015, 4(2): 164-180

Table 11: Inhibitory activity of solvent extracts of selected actinomycetes (PO-10 to PO-16 and PO-18) against test fungi

\begin{tabular}{|c|c|c|c|c|c|c|c|c|}
\hline \multirow{2}{*}{ Isolate } & \multirow{2}{*}{$\begin{array}{l}\text { Solvent } \\
\text { extract }\end{array}$} & \multicolumn{7}{|c|}{ Test fungi/Zone of inhibition in $\mathrm{cm}($ Mean $\pm S D)$} \\
\hline & & C. albicans & C. neoformans & A. niger & A. flavus & P. notatum & R. stolonifer & F. oxysporum \\
\hline \multirow{3}{*}{ PO-10 } & $\mathrm{H}$ & $0.8 \pm 0.0$ & $0.8 \pm 0.0$ & $0.8 \pm 0.0$ & $0.8 \pm 0.0$ & $0.0 \pm 0.0$ & $0.0 \pm 0.0$ & $0.8 \pm 0.0$ \\
\hline & EA & $1.2 \pm 0.0$ & $1.3 \pm 0.0$ & $0.8 \pm 0.0$ & $1.0 \pm 0.0$ & $0.8 \pm 0.0$ & $0.8 \pm 0.0$ & $1.2 \pm 0.2$ \\
\hline & $\mathrm{B}$ & $1.0 \pm 0.0$ & $0.8 \pm 0.0$ & $0.8 \pm 0.0$ & $0.8 \pm 0.0$ & $0.0 \pm 0.0$ & $0.0 \pm 0.0$ & $0.8 \pm 0.0$ \\
\hline \multirow{3}{*}{ PO-11 } & $\mathrm{H}$ & $0.0 \pm 0.0$ & $0.0 \pm 0.0$ & $0.0 \pm 0.0$ & $0.0 \pm 0.0$ & $0.0 \pm 0.0$ & $0.0 \pm 0.0$ & $0.0 \pm 0.0$ \\
\hline & EA & $0.0 \pm 0.0$ & $0.0 \pm 0.0$ & $0.0 \pm 0.0$ & $0.0 \pm 0.0$ & $0.0 \pm 0.0$ & $0.0 \pm 0.0$ & $0.8 \pm 0.0$ \\
\hline & $\mathrm{B}$ & $0.8 \pm 0.0$ & $1.0 \pm 0.0$ & $0.8 \pm 0.0$ & $0.0 \pm 0.0$ & $0.0 \pm 0.0$ & $0.0 \pm 0.0$ & $1.2 \pm 0.0$ \\
\hline \multirow{3}{*}{ PO-12 } & $\mathrm{H}$ & $0.0 \pm 0.0$ & $0.0 \pm 0.0$ & $0.8 \pm 0.0$ & $0.0 \pm 0.0$ & $0.0 \pm 0.0$ & $0.0 \pm 0.0$ & $0.0 \pm 0.0$ \\
\hline & EA & $1.0 \pm 0.0$ & $1.4 \pm 0.2$ & $1.0 \pm 0.0$ & $0.8 \pm 0.0$ & $0.0 \pm 0.0$ & $0.8 \pm 0.0$ & $1.2 \pm 0.0$ \\
\hline & $\mathrm{B}$ & $0.8 \pm 0.0$ & $1.0 \pm 0.2$ & $0.8 \pm 0.0$ & $0.0 \pm 0.0$ & $0.0 \pm 0.0$ & $0.0 \pm 0.0$ & $0.8 \pm 0.0$ \\
\hline \multirow{3}{*}{ PO-13 } & $\mathrm{H}$ & $0.0 \pm 0.0$ & $0.0 \pm 0.0$ & $0.0 \pm 0.0$ & $0.0 \pm 0.0$ & $0.0 \pm 0.0$ & $0.0 \pm 0.0$ & $0.0 \pm 0.0$ \\
\hline & EA & $0.0 \pm 0.0$ & $0.8 \pm 0.0$ & $0.0 \pm 0.0$ & $0.8 \pm 0.0$ & $0.0 \pm 0.0$ & $0.0 \pm 0.0$ & $0.8 \pm 0.0$ \\
\hline & $\mathrm{B}$ & $1.0 \pm 0.0$ & $1.1 \pm 0.1$ & $0.8 \pm 0.0$ & $1.0 \pm 0.1$ & $0.8 \pm 0.0$ & $0.0 \pm 0.0$ & $1.0 \pm 0.1$ \\
\hline \multirow{3}{*}{ PO-14 } & $\mathrm{H}$ & $0.0 \pm 0.0$ & $0.0 \pm 0.0$ & $0.0 \pm 0.0$ & $0.0 \pm 0.0$ & $0.0 \pm 0.0$ & $0.0 \pm 0.0$ & $0.0 \pm 0.0$ \\
\hline & EA & $0.8 \pm 0.0$ & $1.0 \pm 0.0$ & $0.8 \pm 0.0$ & $0.0 \pm 0.0$ & $0.0 \pm 0.0$ & $0.0 \pm 0.0$ & $0.0 \pm 0.0$ \\
\hline & $\mathrm{B}$ & $1.2 \pm 0.2$ & $1.4 \pm 0.2$ & $1.0 \pm 0.0$ & $0.8 \pm 0.0$ & $0.8 \pm 0.0$ & $0.8 \pm 0.0$ & $0.8 \pm 0.0$ \\
\hline \multirow{3}{*}{ PO-15 } & $\mathrm{H}$ & $0.0 \pm 0.0$ & $0.0 \pm 0.0$ & $0.0 \pm 0.0$ & $0.0 \pm 0.0$ & $0.0 \pm 0.0$ & $0.0 \pm 0.0$ & $0.0 \pm 0.0$ \\
\hline & EA & $0.8 \pm 0.0$ & $1.0 \pm 0.0$ & $0.0 \pm 0.0$ & $0.8 \pm 0.0$ & $0.0 \pm 0.0$ & $0.0 \pm 0.0$ & $1.2 \pm 0.0$ \\
\hline & $\mathrm{B}$ & $0.0 \pm 0.0$ & $0.8 \pm 0.0$ & $0.8 \pm 0.0$ & $0.0 \pm 0.0$ & $0.0 \pm 0.0$ & $0.0 \pm 0.0$ & $0.8 \pm 0.0$ \\
\hline \multirow{3}{*}{ PO-16 } & $\mathrm{H}$ & $0.8 \pm 0.0$ & $0.8 \pm 0.0$ & $0.8 \pm 0.0$ & $0.0 \pm 0.0$ & $0.0 \pm 0.0$ & $0.0 \pm 0.0$ & $0.0 \pm 0.0$ \\
\hline & EA & $1.2 \pm 0.0$ & $1.5 \pm 0.1$ & $1.0 \pm 0.0$ & $0.8 \pm 0.0$ & $0.8 \pm 0.0$ & $0.0 \pm 0.0$ & $1.2 \pm 0.0$ \\
\hline & B & $0.8 \pm 0.0$ & $0.8 \pm 0.0$ & $0.8 \pm 0.0$ & $0.0 \pm 0.0$ & $0.0 \pm 0.0$ & $0.0 \pm 0.0$ & $0.8 \pm 0.0$ \\
\hline \multirow{3}{*}{ PO-18 } & $\mathrm{H}$ & $0.0 \pm 0.0$ & $0.0 \pm 0.0$ & $0.8 \pm 0.0$ & $0.0 \pm 0.0$ & $0.0 \pm 0.0$ & $0.0 \pm 0.0$ & $0.0 \pm 0.0$ \\
\hline & EA & $0.8 \pm 0.0$ & $0.8 \pm 0.0$ & $0.0 \pm 0.0$ & $0.8 \pm 0.0$ & $0.0 \pm 0.0$ & $0.0 \pm 0.0$ & $0.8 \pm 0.0$ \\
\hline & $\mathrm{B}$ & $1.1 \pm 0.0$ & $1.2 \pm 0.1$ & $0.0 \pm 0.0$ & $0.8 \pm 0.1$ & $1.0 \pm 0.0$ & $0.0 \pm 0.0$ & $1.0 \pm 0.1$ \\
\hline
\end{tabular}

$\mathrm{H}$ - Hexane; EA- Ethyl acetate; B- Butanol

Brine Shrimp Lethality of Solvent Extracts of Selected Actinomycetes

The result of cytotoxic potential of solvent extracts selected actinomycetes in terms of brine shrimp lethality is shown in Figure 7 and Table 12. At extract concentration of $10 \mu \mathrm{g} / \mathrm{ml}$, none of the extracts caused mortality of brine shrimp larvae. Most extracts showed weaker cytotoxic efficacy. Mortality of larvae at $1000 \mu \mathrm{g} / \mathrm{ml}$ concentration of extracts ranged between 10 and $80 \%$. Among extracts, high cytotoxic potential was observed in case of extract of PO-09 as revealed by lower $I_{50}$ value $(424.44 \mu \mathrm{g} / \mathrm{ml})$. Cytotoxic efficacy of standard $\left(\mathrm{IC}_{50}\right.$ value $(32.25 \mu \mathrm{g} / \mathrm{ml})$ was higher than that of solvent extracts.

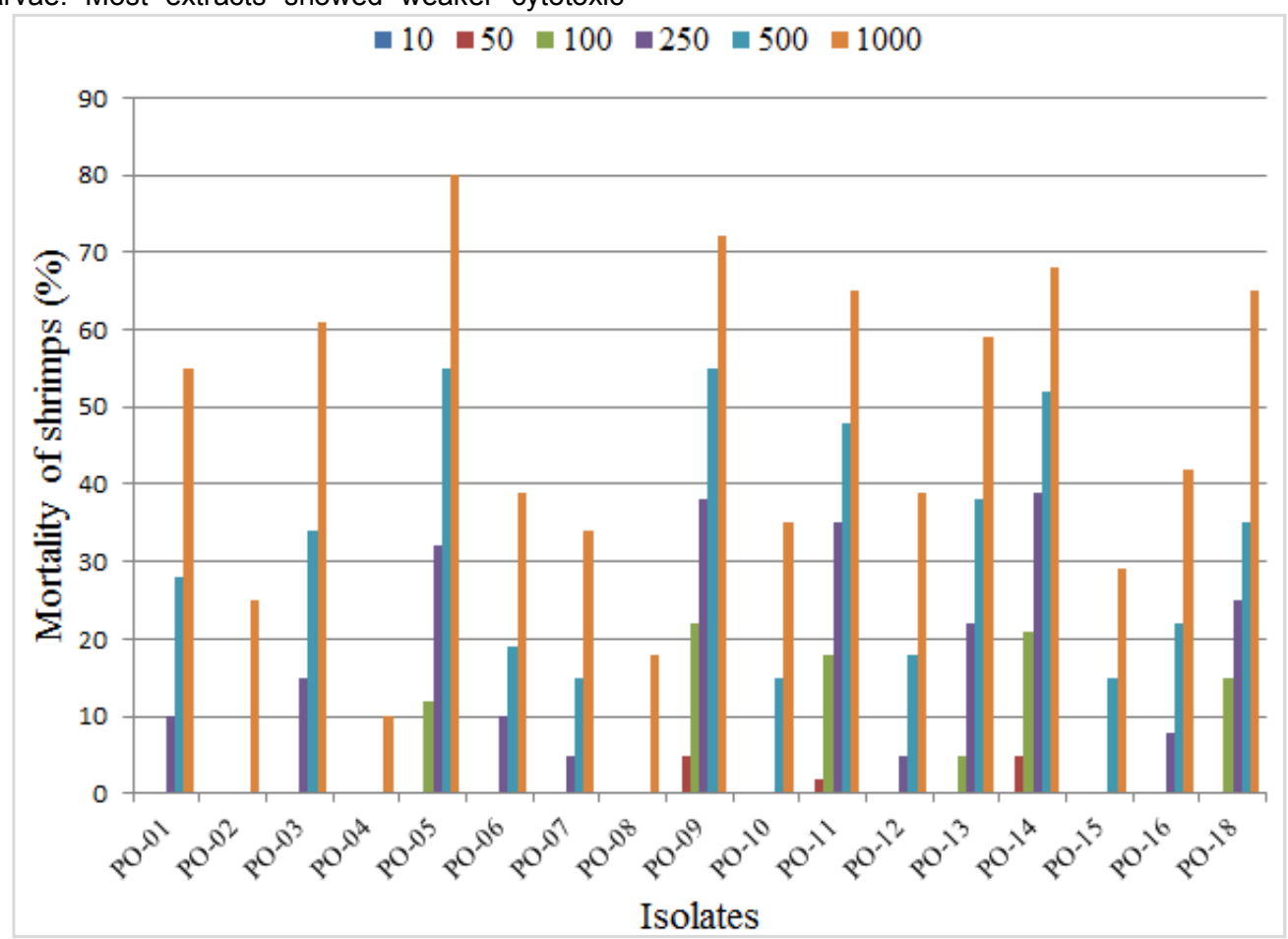

Figure 7: Mortality of brine shrimp larvae by solvent extracts of selected actinomycetes 
Prashith Kekuda et al.,

Table 12: $I_{50}$ values of extracts (brine shrimp lethality assay)

\begin{tabular}{cccc}
\hline Extract & $\mathbf{I C}_{50}(\boldsymbol{\mu g} / \mathbf{m l})$ & Extract & $\mathbf{I C}_{\mathbf{5 0}}(\boldsymbol{\mu g} / \mathbf{m I})$ \\
\hline PO-01 & 906.78 & PO-10 & 1350.00 \\
PO-02 & - & PO-11 & 487.39 \\
PO-03 & 808.33 & PO-12 & 1261.36 \\
PO-04 & - & PO-13 & 796.51 \\
PO-05 & 447.72 & PO-14 & 441.44 \\
PO-06 & 1315.79 & PO-15 & 1761.93 \\
PO-07 & 1434.21 & PO-16 & 1181.82 \\
PO-08 & - & PO-18 & 743.43 \\
PO-09 & 424.44 & Standard & 32.25 \\
\hline
\end{tabular}

Antioxidant Activity of Solvent Extracts of Selected Actinomycetes

DPPH radical scavenging activity of solvent extracts

The result of DPPH radical scavenging potential of solvent extracts of selected actinomycetes is shown in Table 13 and Figure 8 . The solvent extracts exhibited concentration dependent scavenging of DPPH radicals i.e., scavenging efficacy increased on increasing concentration of extracts. At concentration $10 \mu \mathrm{g} / \mathrm{ml}$, none

Sci. Technol. Arts Res. J., April-June 2015, 4(2): 164-180

of the extracts showed scavenging activity. At concentration $200 \mu \mathrm{g} / \mathrm{ml}$, the scavenging potential of extracts ranged between 15.51 to $34.14 \%$. Among extracts tested, extract of PO-12 showed marked scavenging potential as indicated by lower $\mathrm{IC}_{50}$ value $(309.61 \mu \mathrm{g} / \mathrm{ml})$. Least scavenging effect was exhibited by extract of isolate PO-05 $\left(\mathrm{IC}_{50}\right.$ value $\left.763.77 \mu \mathrm{g} / \mathrm{ml}\right)$. The $\mathrm{IC}_{50}$ value for extracts ranged between 309.61 to $763.77 \mu \mathrm{g} / \mathrm{ml}$. Reference antioxidant i.e., ascorbic acid exhibited stronger scavenging activity $\left(\mathrm{IC}_{50}\right.$ value $5.41 \mu \mathrm{g} / \mathrm{ml}$ ) when compared to solvent extracts.

Table 13: IC $\mathrm{I}_{50}$ values (DPPH assay) of solvent extracts

\begin{tabular}{cccc}
\hline Extract & $\mathbf{I C}_{\mathbf{5 0}}(\boldsymbol{\mu} \mathbf{g} / \mathbf{m l})$ & Extract & $\mathbf{I C}_{\mathbf{5 0}}(\boldsymbol{\mu} \mathbf{g} / \mathbf{m I})$ \\
\hline PO-01 & 591.69 & PO-10 & 763.27 \\
PO-02 & 367.24 & PO-11 & 353.20 \\
PO-03 & 520.91 & PO-12 & 309.61 \\
PO-04 & 469.47 & PO-13 & 575.54 \\
PO-05 & 763.77 & PO-14 & 634.61 \\
PO-06 & 472.49 & PO-15 & 339.53 \\
PO-07 & 515.71 & PO-16 & 627.97 \\
PO-08 & 352.42 & PO-18 & 479.70 \\
PO-09 & 661.83 & Standard & 5.41 \\
\hline
\end{tabular}

$\square=10 \square 25 \square 50 \square 100 \square 200$

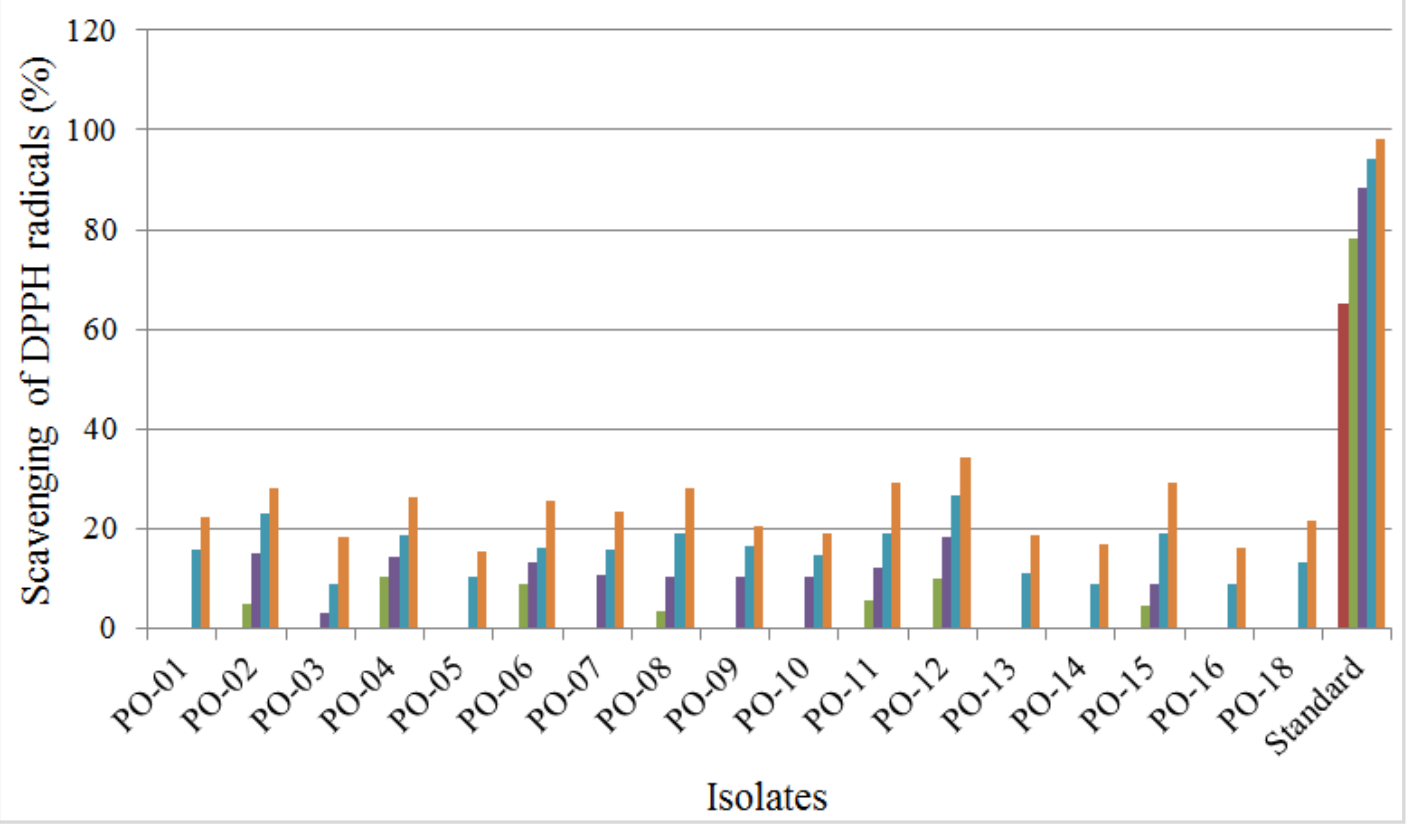

Figure 8: Scavenging of DPPH radicals by solvent extracts

ABTS Radical Scavenging Activity of Solvent Extracts The result of ABTS radical scavenging potential of solvent extracts is shown in Table 14 and Figure 9. The extracts displayed dose dependent scavenging of ABTS radicals. At concentration $10 \mu \mathrm{g} / \mathrm{ml}$, none of the extracts exhibited scavenging potential. At concentration $200 \mu \mathrm{g} / \mathrm{ml}$, the scavenging potential of extracts ranged between 16.65 to $38.68 \%$. The $I C_{50}$ value for extracts ranged between 275.08 to $645.54 \mu \mathrm{g} / \mathrm{ml}$. Among extracts, extract of PO-10 caused high scavenging of radicals as revealed by lower $I C_{50}$ value $(275.08 \mu \mathrm{g} / \mathrm{ml})$. Extract of PO-03 showed least scavenging effect on radicals $\left(\mathrm{IC}_{50}\right.$ value $645.54 \mu \mathrm{g} / \mathrm{ml}$ ). Reference antioxidant displayed higher scavenging potential $\left(\mathrm{IC}_{50}\right.$ value $\left.6.88 \mu \mathrm{g} / \mathrm{ml}\right)$ than solvent extracts.
Table 14: $I C_{50}$ values (ABTS assay) of solvent extracts

\begin{tabular}{cccc}
\hline Extract & $\mathbf{I C}_{\mathbf{5 0}}(\boldsymbol{\mu} \mathbf{g} / \mathrm{ml})$ & Extract & $\mathbf{I C}_{\mathbf{5 0}}(\boldsymbol{\mu} \mathbf{g} / \mathbf{m l})$ \\
\hline PO-01 & 311.44 & PO-10 & 275.08 \\
PO-02 & 478.77 & PO-11 & 375.03 \\
PO-03 & 645.54 & PO-12 & 432.09 \\
PO-04 & 571.10 & PO-13 & 482.26 \\
PO-05 & 297.90 & PO-14 & 553.64 \\
PO-06 & 403.31 & PO-15 & 537.16 \\
PO-07 & 595.24 & PO-16 & 344.89 \\
PO-08 & 361.30 & PO-18 & 403.88 \\
PO-09 & 360.57 & Standard & 6.88 \\
\hline
\end{tabular}




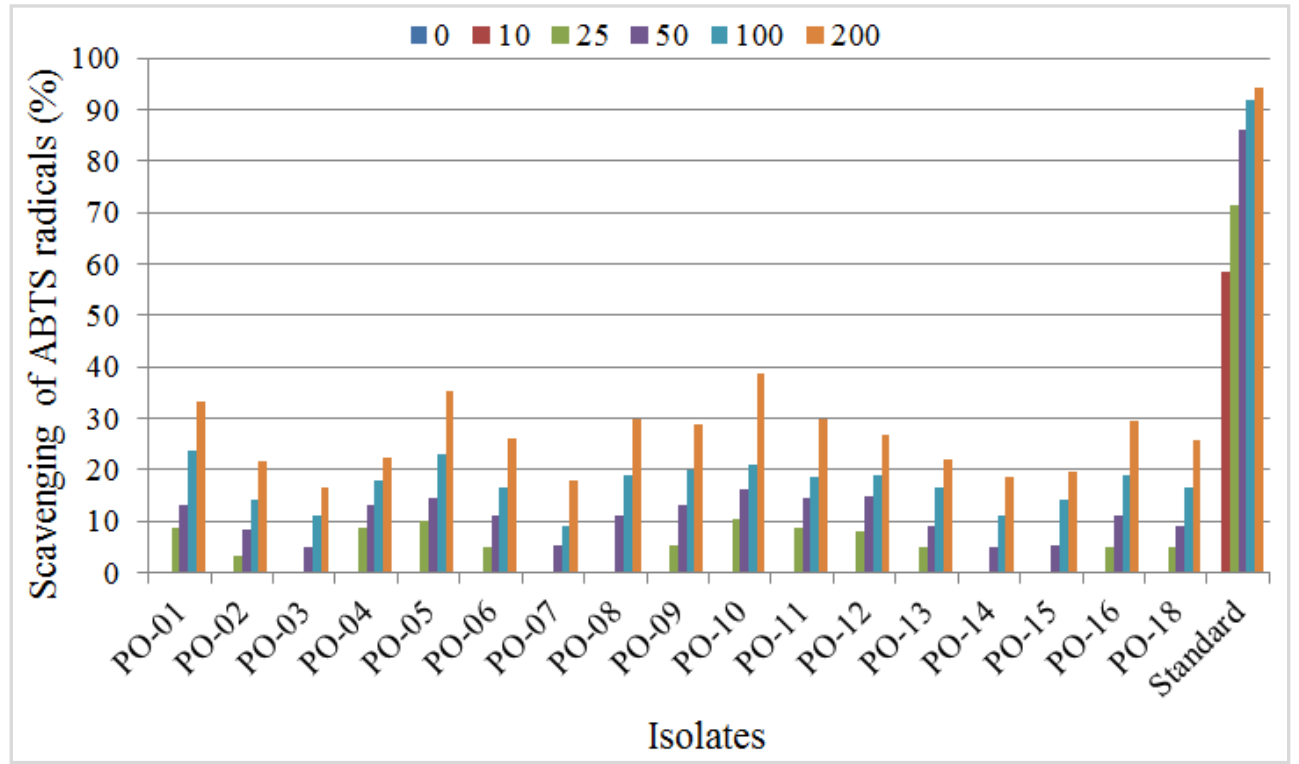

Figure 9: Scavenging of ABTS radicals by solvent extracts of selected actinomycetes

\section{Metal chelating activity of solvent extracts}

Table 15 and Figure 10 shows the result of metal chelating effect of solvent extracts. The extracts displayed concentration dependent chelating activity. No chelating activity was observed at $10 \mu \mathrm{g} / \mathrm{ml}$ concentration of all extracts. At concentration $200 \mu \mathrm{g} / \mathrm{ml}$, the chelating potential of extracts ranged between 12.36 and $22.06 \%$. The $I C_{50}$ value for extracts ranged between 529.36 and $961.22 \mu \mathrm{g} / \mathrm{ml}$. Among solvent extracts, extract of PO-03 displayed higher activity $\left(\mathrm{IC}_{50}\right.$ value $\left.529.36 \mu \mathrm{g} / \mathrm{ml}\right)$ and extract of PO-13 showed least chelating activity (IC $\mathrm{IC}_{50}$ value $961.22 \mu \mathrm{g} / \mathrm{ml})$. Chelating effect of reference standard $\left(\mathrm{IC}_{50}\right.$ value $9.29 \mu \mathrm{g} / \mathrm{ml}$ ) was higher than solvent extracts.
Table 15: $\mathrm{IC}_{50}$ values (Metal chelating activity) of solvent extracts

\begin{tabular}{cccc}
\hline Extract & $\mathbf{I C}_{\mathbf{5 0}}(\boldsymbol{\mu} \mathbf{g} / \mathbf{m l})$ & Extract & $\mathbf{I C}_{\mathbf{5 0}}(\boldsymbol{\mu} \mathbf{g} / \mathbf{m l})$ \\
\hline PO-01 & 580.83 & PO-10 & 541.23 \\
PO-02 & 722.68 & PO-11 & 573.92 \\
PO-03 & 529.36 & PO-12 & 718.64 \\
PO-04 & 545.54 & PO-13 & 961.22 \\
PO-05 & 786.75 & PO-14 & 571.53 \\
PO-06 & 658.87 & PO-15 & 531.05 \\
PO-07 & 571.77 & PO-16 & 529.55 \\
PO-08 & 551.71 & PO-18 & 541.69 \\
PO-09 & 617.38 & Standard & 9.29 \\
\hline
\end{tabular}

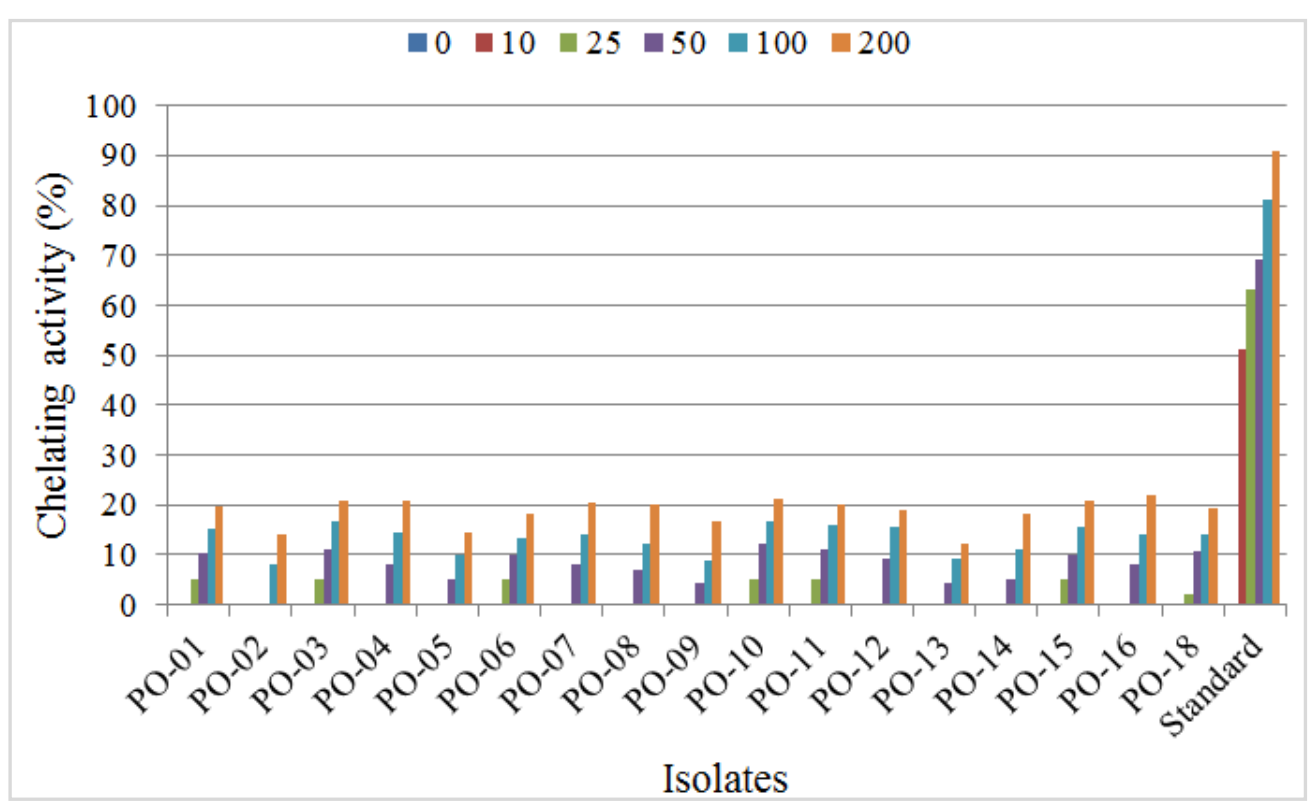

Figure 10: Metal chelating activity of solvent extracts of selected actinomycetes

Ferric Reducing Activity of Solvent Extracts

Figure 11 shows the result of ferric reducing activity of solvent extracts and reference standard. The absorbance of reaction mixtures increased on increasing the concentration of extract. Among extracts, extract of PO-10 displayed higher reducing activity. Reducing potential of ascorbic acid was greater than that of solvent extracts of actinomycetes. 


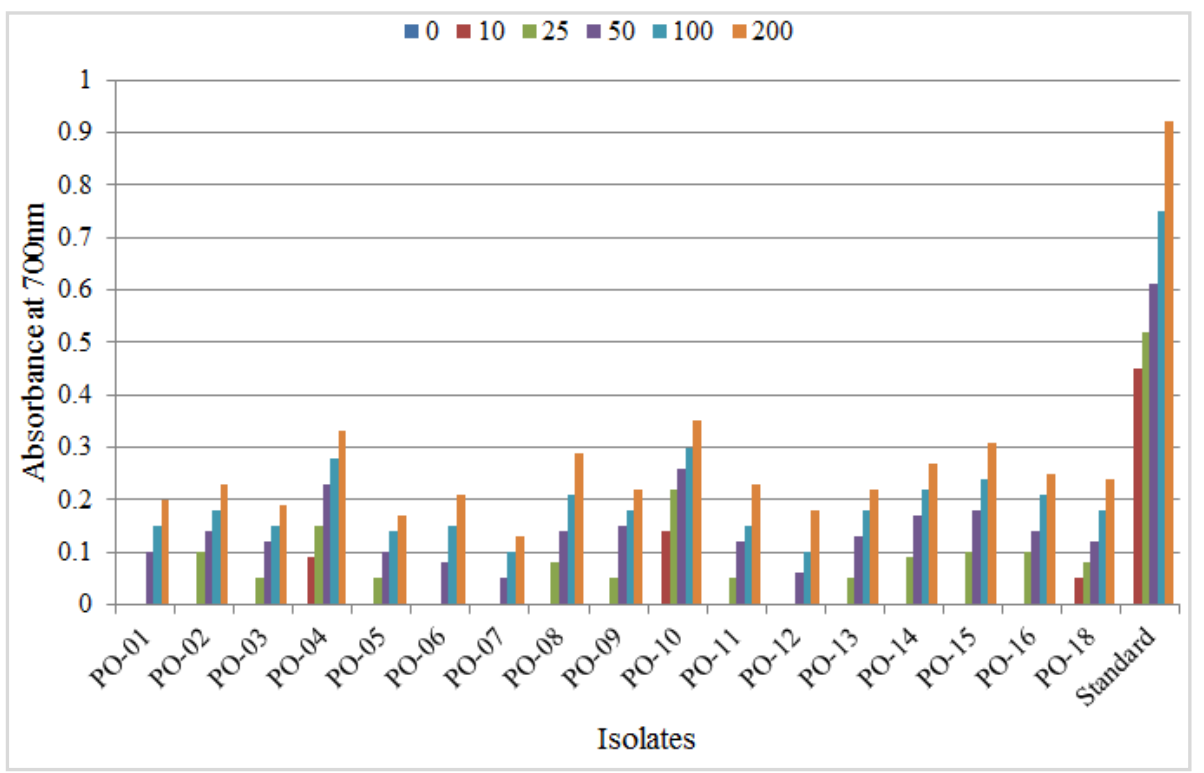

Figure 11: Ferric reducing activity of solvent extracts

\section{DISCUSSION}

Soil is inhabited by a large number of organisms. The rhizosphere is a unique biological niche present in vicinity of plant roots. Rhizosphere is profoundly influenced by exudates released from plant roots and the microbial community is nutritionally favored by root exudates. Many of soil microbes are useful as they produce a number of bioactive metabolites, including clinically important antibiotics. Actinomycetes are abundant in soil, species of Streptomyces in particular. Streptomycetes represent the dominant actinomycetes genera in soil and play an important role in recycling of materials and production of bioactive compounds (Ceylan et al., 2008; Gautham et al., 2012; Junaid et al., 2013; Kekuda et al., 2013b). A variety of media such as Glycerol-Yeast extract agar, Starch casein agar, inorganic salts starch agar, Humic acid Vitamin agar, Kuster's agar, Yeast extract-malt extract agar, Oat meal agar, Actinomycetes isolation agar and Glycerol aspargine agar are used for isolation of actinomycetes. SCN agar is one of the most commonly used media for isolation of actinomycetes from a variety of samples (Xu et al., 1996; Moncheva et al., 2000-2002; Rifaat, 2003; Anansiriwattana et al., 2006; Gautham, 2012; Kekuda et al., 2013a). The present study concentrated on isolating actinomycetes from different places of Western Ghat of Shivamogga. A total of 57 soil samples were collected from 13 places. A total of 182 actinomycetes isolates were recovered on SCN agar.

The primary screening for antagonistic efficacy of isolated actinomycetes was done by using cross streak and dual inoculation technique. The primary screening revealed broad and narrow spectrum antimicrobial activity of the isolates. On the basis of the result of primary screening, 17 isolates were chosen for further studies as they exhibited marked inhibitory activity against bacteria and fungi. Cross streak method is one of the widely used preliminarily screening methods to detect the possible antagonistic potential of actinomycetes isolates. This technique forms the basis to delineate potent isolates for secondary screening procedures. The absence of growth or a less dense growth of test organisms near the actinomycetes isolates was considered positive for production and secretion of antimicrobial metabolite by the isolates (Haque et al., 1996; Sahin and Ugur, 2003; Nanjwade et al., 2010; Dasari et al., 2011; Gautham et al., 2012). The isolates namely PO-05, PO-10 and PO-13 performed well in primary screening for antibacterial activity. Isolates $\mathrm{PO}-05$ and $\mathrm{PO}-11$ inhibited fungi to maximum extent.

In this study, 17 actinomycetes isolates showing marked antimicrobial effect in primary screening were characterized on the basis of cultural, microscopic, staining and biochemical characteristics. The colonies of isolates exhibited appearance such as raised, powdery, rugose, radiating, discrete and velvety being characteristic of actinomycetes. The colors of aerial mycelia and substrate mycelia varied among isolates. Most of the isolates belonged to grey series. Spore arrangements of various kinds viz., spiral, loop, straight, hook and flexibilis were observed. All isolates were Gram positive and non acid-fast. The isolates were biochemically versatile in terms of utilization of various sources. On the basis of cultural characteristics, microscopic features together with biochemical characteristics, all 17 isolates were found to be species of the genus Streptomyces. Morphology plays an important role in distinguishing Streptomyces from other sporing actinomycetes and in the characterization of Streptomycete species. The 3 features viz., vegetative mycelium, aerial mycelium baring chains of spores and the characteristics of spores themselves are key for identification of Streptomycetes. The latter two features are considered most diagnostic information (Anderson and Wellington, 2001; Taddei et al., 2006). Information on cultural features and characteristic spore arrangement together with biochemical properties assists classification of actinomycetes as members of the genus Streptomyces (Cao et al., 2004; Oskay et al., 2004; Laidi et al., 2006; Gautham et al., 2012; Kekuda et al., 2013a).

Solvent extracts of actinomycetes, in particular Streptomyces species, exhibit antimicrobial activity (Augustine et al., 2005; Taechowisan et al., 2005; Gautham, 2012; Kekuda et al., 2013a). In the present study, the bioactive isolates were grown in SCN broth and the culture filtrate was extracted using solvents viz., hexane, ethyl acetate and butanol. The solvent extracts 
were screened for antimicrobial activity by agar well diffusion assay. Overall, ethyl acetate was found to be the best solvent for extraction of bioactive metabolites as ethyl acetate extract of most of the isolates caused marked inhibitory activity against test bacteria and test fungi. Next to ethyl acetate, butanol extract exhibited stronger inhibitory activity against bacteria and fungi. Least inhibitory effect was observed against test microbes by hexane extracts of isolates. It has been shown that various solvents are effectively used to extract bioactive metabolites by actinomycetes. Solvents of different polarity such as butanol, ethyl acetate, acetone, hexane, ethanol and methanol have been shown to extract metabolites efficiently (Sahin and Ugur, 2003; Augustine et al., 2005; Gautham, 2012; Kekuda et al., 2013a).

It has been shown that Western Ghat actinomycetes of Karnataka exhibit antimicrobial activity. The Streptomyces species from Agumbe (Kekuda et al., 2012; Gautham et al., 2012), Streptomyces species RAMPP-065 from Kudremukh (Manasa et al., 2012) and Streptomyces species SRDP-07 from Thirthahalli (Kekuda et al., 2013) exhibited antibacterial activity. In the present study, it has been observed that Gram positive bacteria, overall, showed higher susceptibility than Gram negative bacteria to solvent extracts of selected actinomycetes. Similar results were observed in the studies of Kekuda et al. (2012), Manasa et al. (2012), Kekuda et al. (2013) and Kekuda and Onkarappa (2015) which reported stronger inhibitory activity of extracts of Streptomyces species against Gram positive bacteria when compared to Gram negative bacteria. The low antibacterial activity of extracts of selected actinomycetes against the Gram negative bacteria could be ascribed to the presence of an outer membrane that possess hydrophilic polysaccharides chains and forms an additional barrier for extract as well as antibiotics (Lodhia et al., 2009; Nalubega et al., 2011). In earlier studies, Streptomyces species from Agumbe (Gautham et al., 2012) and Kodachadri (Shobha and Onkarappa, 2011) were shown to exhibit antifungal activity. In the present study, the Streptomyces species from various places of Western Ghats of Shivamogga district displayed antifungal activity against yeasts and molds. Antimicrobial activity of purified compounds from Western Ghat actinomycetes has been investigated. In an earlier study, Gautham (2012) isolated xyloglucose disaccharide antibiotic from Streptomyces fradiae and showed that the antibiotic had activity against bacteria and fungi. More recently, Kekuda et al. (2014b) recovered a glycoside compound from Streptomyces variabilis PO178 and showed its inhibitory activity against Gram positive and Gram negative bacteria.

In the present study, the cytotoxic nature of solvent extracts of selected actinomycetes was checked by brine shrimp lethality bioassay. The assay is in vivo and employs a simple zoologic organism as a convenient monitor for screening, discovering and monitoring various bioactivities of various samples. This test is more useful in determining bioactivities such as cytotoxic, phototoxic, pesticidal, trypanocidal, enzyme inhibition and ion regulation activities. This assay can also be extrapolated for cell-line toxicity and antitumor activity. The method is simple, rapid as it utilizes only 24 hours, inexpensive, needs no special equipment and does not require aseptic conditions. The assay employs large number of organisms for validation and a relatively small amount of sample (McLaughlin, 1991; Luis et al., 2002; Hossain et al., 2009). It has been shown that solvent extracts and purified compounds from actinomycetes possess cytotoxic effect in terms of brine shrimp lethality effect (Sultan et al., 2002; Manivasagan et al., 2009; Kesavan et al., 2011; Sosovele et al., 2012; Kekuda et al., 2012; Sharmin et al., 2013). In this study, the solvent extracts of selected actinomycetes exhibited dose dependent cytotoxicity with $\mathrm{LC}_{50}$ ranging between 424.44 and $1761 \mu \mathrm{g} / \mathrm{ml}$. Potent cytotoxicity was observed in case isolate PO-09. In similar studies, Kekuda et al. (2011) and Kekuda et al. (2012) and Kekuda and Onkarappa (2015) observed dose dependent mortality of brine shrimp larvae by extracts of a Streptomyces species isolated from Agumbe and their cytotoxic effect was higher than that of result of present study.

Among various in vitro radical scavenging assays, the DPPH free radical scavenging assay is widely used to evaluate antioxidant capacities of various kinds of samples including solvent extracts or purified compounds from actinomycetes. DPPH is an organic, stable, nitrogen centred, purple colored free radical exhibiting absorption maximum around $515-528 \mathrm{~nm}$ in alcoholic solution. The radical becomes a stable diamagnetic molecule upon accepting an electron or hydrogen atom. Antioxidants react with $\mathrm{DPPH}$, transfers proton and results in decolorization of radical (color change from purple to yellow). The degree of discoloration depends on the ability of antioxidant compound to donate hydrogen proton (Braca et al., 2001; Sharma and Bhat, 2009; Kekuda et al., 2010b; Sowndhararajan and Kang, 2013; Kekuda et al., 2013; Subathradevi et al., 2014; Lee et al., 2014). In this study, the absorbance of DPPH in the presence of various concentrations of solvent extracts of selected actinomycetes was measured at $517 \mathrm{~nm}$. The scavenging of DPPH radicals was dose dependent. Extract of PO-12 and PO-05 displayed highest and least scavenging of radicals. It was observed that the scavenging efficacy of solvent extracts was lesser than that of ascorbic acid. Similar observations were reported in earlier studies of Kekuda et al. (2010b), Manasa et al. (2012), Kekuda et al. (2013), Gautham and Onkarappa (2013) and Junaid et al. (2013) in which Streptomyces species from different parts of Western Ghats of Karnataka exhibited low to moderate scavenging of DPPH radicals. Although the scavenging abilities of extracts were lesser than that of ascorbic acid, it was evident that the solvent extracts showed hydrogen donating ability and the extract could serve as free radical scavengers, acting possibly as primary antioxidants (Chung et al., 2006). Several other studies have also revealed the potential of actinomycetes to scavenge DPPH radicals (Sowndhararajan and Kang, 2013; Subathradevi et al., 2014; Lee et al., 2014).

Similar to DPPH assay, ABTS radical scavenging is another popular in vitro assay which measures the radical scavenging nature of several types of compounds. On interaction with ABTS, compounds (antioxidants) either transfer electrons or hydrogen atoms to ABTS and thereby neutralizing its free radical character ( $R e$ et al., 1999; Zhong et al., 2011; Li et al., 2011; Sowndhararajan and Kang, 2013; Lee et al., 2014). ABTS is converted to its radical cation by reacting it with sodium persulfate. The ABTS radical is blue in color and absorbs light at $730 \mathrm{~nm}$. The ABTS radical cation can react with most antioxidants. On reaction, the blue ABTS radical cation is converted into colorless. The extent of reaction is monitored spectrophotometrically. In this study, we evaluated free 
Prashith Kekuda et al.,

radical scavenging effect of solvent extracts by $A B T S$ assay. The scavenging of radicals was dose dependent. Extract of $\mathrm{PO}-10$ and $\mathrm{PO}-03$ showed high and least scavenging efficacy. Ascorbic acid displayed higher scavenging potential than solvent extracts. Crude solvent extracts and purified compounds from actinomycetes were shown to exhibit ABTS radical scavenging activity. Extract of Streptomyces strain Eri12 (Zhong et al., 2011) and Streptomyces sp. Strain MJM10778 (Lee et al., 2014) scavenged ABTS radicals. Novobiocin isolated from halophilic actinomycete Nocardiopsis gilva YIM 90087 displayed moderate scavenging of ABTS radicals (Tian et al., 2013).

Iron can stimulate lipid peroxidation by the Fenton reaction, and also accelerates peroxidation by decomposing lipid hydroperoxides into peroxyl and alkoxyl radicals that can themselves abstract hydrogen and perpetuate the chain reaction of lipid peroxidation. Metal chelating capacity is important since it reduces the concentration of the catalysing transition metal in lipid peroxidation. It was reported that chelating agents form bonds with a metal and are effective as secondary antioxidants because they reduce the redox potential thereby stabilizing the oxidized form of the metal ion. Ferrozine can quantitatively form complexes with $\mathrm{Fe}^{2+}$. In the presence of chelating agents, the complex formation is disrupted and leads to fading of the red color of the complex (Hseu et al., 2008; Jindal and Mohamad, 2012). In this study, the formation of the $\mathrm{Fe}^{2+}$-ferrozine complex was not completed in the presence of solvent extracts of actinomycetes, indicating the potential of solvent extracts to chelates the iron and capture ferrous ion before ferrozine. It was observed that the absorbance of $\mathrm{Fe}^{2+}$. ferrozine complex decreased dose-dependently. Extract of $\mathrm{PO}-03$ and $\mathrm{PO}-13$ displayed high and least chelating effect. The data obtained in this study reveals that the solvent extracts demonstrate capacity for iron binding, suggesting that their action as peroxidation protector may be related to its iron binding capacity. However, the chelating effect was weaker when compared to reference agent. Similar results were obtained in an early study of Gautham and Onkarappa (2013) where extract of Streptomyces fradiae GOS1 isolated from Agumbe showed weaker chelating potential.

In this study, the measurement of direct reduction of $\mathrm{Fe}^{+3}(\mathrm{CN})_{6}$ to $\mathrm{Fe}^{+2}(\mathrm{CN})_{6}$ was employed in order to evaluate the reducing power of solvent extracts of selected actinomycetes. It is determined by measuring the absorbance resulting from the formation of Perl's Prussian blue complex on addition of excess of ferric ions $\left(\mathrm{Fe}^{+3}\right)$. An increase in absorbance at $700 \mathrm{~nm}$ on increase in extract concentrations indicates reducing capacity and the reducing properties are generally associated with the presence of reductones. Ferric reducing assay has been employed to evaluate antioxidant activity of compounds. In this assay, the presence of reductants (antioxidants) in the samples would result in the reduction of $\mathrm{Fe}^{+3}$ to $\mathrm{Fe}^{+2}$ by donating an electron. The reducing capacity of a compound may serve as a significant indicator of its potential antioxidant activity (Hsu et al., 2006; Chung et al., 2006; Kekuda et al., 2013a). In this study, the solvent extracts of selected actinomycetes showed dose dependent reducing power and the effect was lesser when compared to ascorbic acid. Overall, the reducing power of extract of isolate $\mathrm{PO}-10$ was marked when compared to solvent extracts of other isolates. Although the effect observed is lesser, it is evident that the extracts
Sci. Technol. Arts Res. J., April-June 2015, 4(2): 164-180

possess reductive potential and could serve as electron donors, terminating the radical chain reactions (Chung et al., 2006). Similar results were observed in earlier studies on Western Ghat actinomycetes in which solvent extracts of Streptomyces species isolated from Thirthahalli (Kekuda et al., 2013), Agumbe (Kekuda et al., 2010b; Gautham and Onkarappa, 2013), Kudremukh (Manasa et al., 2012) and Talakaveri (Junaid et al., 2013) displayed low to moderate ferric reducing activity.

\section{CONCLUSION}

New screening programs have been developed for isolation of new species and novel and biologically active compounds. One such approach is to isolate and screen actinomycetes from relatively unknown or unstudied areas. In the present study, we isolated Streptomyces species from different soil samples of Western Ghats of Karnataka. The isolates have shown antimicrobial, antioxidant and cytotoxic efficacies. The Western Ghat actinomycetes are found to be promising resources for the development of therapeutic agent. Further studies on isolation of active components from the isolates and their antimicrobial and cytotoxic activity determination are to be carried out.

\section{Conflict of Interest}

Conflict of interest none declared.

\section{Acknowledgements}

Authors are thankful to Principal, Sahyadri Science College (Autonomous), Kuvempu University for providing facilities and moral support to conduct work. Authors also thank Dr. N. Mallikarjun \& Dr. Mukunda S for their support

\section{REFERENCES}

Aiyegoro, O.A., Okoh, A.I. (2010). Preliminary phytochemical screening and in vitro antioxidant activities of the aqueous extract of Helichrysum longifolium DC. BMC Complementary and Alternative Medicine 10: 21.

Alimuddin, Widada, J., Asmara, W., Mustofa. (2011). Antifungal production of a strain of Actinomycetes spp isolated from the rhizosphere of Cajuput plant: Selection and detection of exhibiting activity against tested fungi. Indonesian Journal of Biotechnology 16(1): 1-10.

Anansiriwattana, W., Tanasupawat, S., Amnuoypol, S., Suwanborirux, K. (2006). Identification and antimicrobial activities of actinomycetes from soils in Samed Island, and geldamycin from strain PC4-3. Thai Journal of Pharmaceutical Sciences 30: 49-56.

Anderson, A.S., Wellington, E.M.H. (2001). The taxonomy of Streptomyces and related genera. International Journal of Systematic and Evolutionary Microbiology 51: 797-814.

Aneja, K.R. (1996). Experiments in Microbiology, Plant pathology, Tissue culture and Mushroom cultivation. $2^{\text {nd }}$ Edition. Vishwa Prakashan, New Delhi

Augustine, S.K., Bhavsar, S.P., Kapadnis, B.P. (2005). A non-polyene antifungal antibiotic from Streptomyces albidoflavus PU23. Journal of Biosciences 30(2):201-211.

Braca, A., De Tommasi, N., Di Bari, L., Pizza, C., Politi, M., Morelli, I. (2001). Antioxidant principles from Bauhinia tarapotensis. Journal of Natural Products 64(7): 892-895.

Cao, L., Qiu, Z., Dai, X., Tan, H., Lin, Y., Zhou, S. (2004). Isolation of endophytic actinomycetes from roots and leaves of banana (Musa acuminata) plants and their activities against Fusarium oxysporum f. sp. cubense. World Journal of Microbiology \& Biotechnology 20:501-04

Cappuccino, J.G., Sherman, N. (1999). Microbiology- A laboratory manual. $4^{\text {th }}$ Edi. Addison Wesley Longman Inc. 
Prashith Kekuda et alo,

Ceylan, O., Okmen, G., Ugur, A. (2008). Isolation of soil Streptomyces as source of antibiotics active against antibiotic-resistant bacteria. EurAsian Journal of Biosciences 2: 73-82.

Chang, H., Ho, Y., Sheu, M., Lin, Y., Tseng, M., Wu, S., Huang, G., Chang, Y. (2007). Antioxidant and free radical scavenging activities of Phellinus merrillii extracts. Botanical Studies 48: 407-417.

Chung, Y., Chien, C., Teng, K., Chou, S. (2006). Antioxidative and mutagenic properties of Zanthoxylum ailanthoides Sieb and zucc. Food Chemistry 97: 418-425.

Dasari, V.R.R.K., Nikku, M.Y., Donthireddy, S.R.R. (2011). Screening of Antagonistic Marine Actinomycetes: Optimization of Process Parameters for the Production of Novel Antibiotic by Amycolatopsis alba var. nov. DVR D4. Journal of Microbial and Biochemical Technology 3:5.

Davies, J., Davies, D. (2010). Origins and evolution of antibiotic resistance. Microbiology and Molecular Biology Reviews 74(3): 417-433.

de Mesquita, M.L., de Paula, J.E., Pessoa, C., de Moraes, M.O., Costa-Lotufo, L.V., Grougnet, R., Michel, S. Tillequin, F., Espindola, L.S. (2009). Cytotoxic activity of Brazilian Cerrado plants used in traditional medicine against cancer cell lines. Journal of Ethnopharmacology 123(3): 439-45.

Dinis, T.C.P., Madeira, V.M.C., Almedia, L.M. (1994). Action of phenolic derivatives (acetaminophen, salicylate and 5aminosalicylate) as inhibitors of membrane lipid peroxidation and phenolic radical scavengers. Achieves in Biochemistry and Biophysics 315(1): 161-169.

Ebrahimzadeh, M.A., Nabavi, S.M., Nabavi, S.F., Esmai, B. (2010). Antioxidant activity of the bulb and aerial parts of Ornithogalum sintenisii L (Liliaceae) at flowering Stage. Tropical Journal of Pharmaceutical Research 9(2):141-48

Gautham, S.A. (2012). Molecular characterization and pharmacological activities of metabolites from Streptomyces spp. Ph.D thesis. Kuvempu University, Karnataka, India.

Gautham, S.A., Onkarappa, R. (2013). In vitro antioxidant activity of metabolite from Streptomyces fradiae strain GOS1. International Journal of Drug Development and Research 5(1): 235-244.

Gautham, S.A., Onkarappa, R. (2013). Pharmacological activities of metabolite from Streptomyces fradiae strain GOS1. International Journal of Chemical Sciences 11(1): 583-590.

Gautham, S.A., Shobha, K.S., Onkarappa, R., Kekuda, P.T.R. (2012). Isolation, characterization and antimicrobial potential of Streptomyces species from Western Ghats of Karnataka, India. Research Journal of Pharmacy and Technology 5(2): 233-238.

Haque, S.K., Sen, S.K., Pal, S.C. (1996). Antimicrobial spectra and toxicity of antibiotics from Streptomyces antibioticus SR 15-4. Indian Journal of Microbiology 36: 113-114.

Hossain, A.M., Ferdous, T., Salehuddin, S.M., Das, A.K. (2009). In vitro cytotoxicity $\left(\mathrm{LC}_{50}\right)$ of extracts obtained from the seeds of Zea mays. Asian Journal of Food AgroIndustry 2(3): 336-34.

Hseu, Y., Chang, W., Chen, C., Liao, L., Huang, C., Lu, F., Chia, Y., Hsu, H., Wu, J., Yang, H. (2008). Antioxidant activities of Toona Sinensis leaves extracts using different antioxidant models. Food \& Chemical Toxicology 46:105114.

Hsu, B., Coupar, I.M., Ng, K. (2006). Antioxidant activity of hot water extract from the fruit of the Doum palm, Hyphaene thebaica. Food Chemistry 98: 317-328.
Sci. Technol. Arts Res. J., April-June 2015, 4(2): 164-180

Junaid, S., Dileep, N., Rakesh, K.N., Kekuda, P.T.R. (2013). Antimicrobial and antioxidant efficacy of Streptomyces species SRDP-TK-07 isolated from a soil of Talakaveri, Karnataka, India. Pharmanest 4(4): 736-750.a

Kekuda, P.T.R., Dileep, N., Junaid, S., Rakesh, K.N., Mesta, S.C., Onkarappa, R. (2013a). Biological activities of Streptomyces species SRDP-07 isolated from soil of Thirthahalli, Karnataka, India. International Journal of Drug Development and Research 5(3): 268-285.

Kekuda, P.T.R., Onkarappa, R. (2014a). Antioxidant, anthelmintic and enzyme inhibitory potential of Streptomyces variabilis strain $\mathrm{PO}-178$ isolated from Western Ghat soil of Agumbe, Karnataka, India. Journal of Biological and Scientific Opinion 2(2): 170-176.

Kekuda, P.T.R., Onkarappa, R. (2015). Biological activities of Streptomyces variabilis strain $\mathrm{PO}-178$ isolated from Western Ghat soil of Agumbe, Karnataka, India. In: Deshmukh, A.M., Jawalikar, J.D., Wakte, P.S (Editors). Current Research in Biochemistry and Microbiology. Oxford Book Company, Delhi, India, pp 166-193.

Kekuda, P.T.R., Onkarappa, R., Jayanna, N.D. (2014b). Characterization and antibacterial activity of a glycoside antibiotic from Streptomyces variabilis PO-178. Science, Technology and Arts Research Journal 3(4): 116-121.

Kekuda, P.T.R., Onkarappa, R., Raghavendra, H.L. (2013b). Pharmacological activities of Streptomyces species PO178 isolated from rhizosphere soil of Agumbe, Karnataka. Science, Technology \& Arts Research Journal 2(2):83-91.

Kekuda, P.T.R., Shobha, K.S., Onkarappa R. (2010b). Studies on antioxidant and anthelmintic activity of two Streptomyces species isolated from Western Ghat soil of Agumbe, Karnataka. Journal of Pharmacy Research 3(1): 26-29.

Kekuda, P.T.R., Shobha, K.S., Onkarappa R. (2011). Pancreatic lipase Inhibitory and cytotoxic potential of a Streptomyces species isolated from Western Ghat soil, Agumbe, Karnataka, India. International Journal of Pharmaceutical and Biological Archives 2(3): 932-937.

Kekuda, P.T.R., Shobha, K.S., Onkarappa, R. (2010a). Fascinating diversity and potent biological activities of Actinomycete metabolites. Journal of Pharmacy Research 3(2): 250-256.

Kekuda, P.T.R., Shobha, K.S., Onkarappa, R., Gautham, S.A., Raghavendra, H.L. (2012). Screening biological activities of a Streptomyces species isolated from soil of Agumbe, Karnataka, India. International Journal of Drug Development and Research 4(3): 104-114.

Kesavan, S.S., Vijayalakshmi, S., Nandhini, U.S., Latha, B.M., Selvam, M.M. (2011). Application of brine shrimp bioassay for screening cytotoxic actinomycetes. International Journal of Pharmacy and Pharmaceutical Science Research 1(3): 104-107.

Kim, B.S., Lee, J.Y., Hwang, B.K. (1998). Diversity of Actinomycetes antagonistic to plant pathogenic fungi in cave and sea-mud soils of Korea. The Journal of Microbiology 36(2): 86-92.

Kokare, C.R., Mahadik, K.R., Kadam, S.S., Chopade, B.A. (2004). Isolation of bioactive marine actinomycetes from sediments isolated from Goa and Maharashtra Coastlines west coast of India). Indian Journal of Marine Sciences 33(3): 248-256.

Kumar, R.S., Rajkapoor, B., Perumal, P. (2011). Antitumor and cytotoxic activities of methanol extract of Indigofera linnaei Ali. Asian Pacific Journal of Cancer Prevention 12: 613-618.

Laidi, R.F., Kansoh, A.L., Elshafei, A.M., Cheikh, B. (2006). Taxonomy, identification and biological activities of a 
Prashith Kekuda et alo,

novel isolate of Streptomyces tendae. Arab Journal of Biotechnology 9(3): 427-436.

Lee, D., Lee, S., Choi, B., Cheng, J., Lee, Y., Yang, S.H., Suh, J. (2014). Antioxidant activity and free radical scavenging activities of Streptomyces sp. Strain MJM10778. Asian Pacific Journal of Tropical Medicine 7(12): 962-967.

Li, P., Huo, L., Su, W., Lu, R., Deng, C., Liu, L., Deng, Y., Guo, N., Lu, C., He, C. (2011). Free radical-scavenging capacity, antioxidant activity and phenolic content of Pouzolzia zeylanica. Journal of Serbian Chemical Society 2011; 76 (5): 709-717.

Lodhia, M.H., Bhatt, K.R., Thaker, V.S. (2009). Antibacterial activity of essential oils from Palmarosa, Evening Primrose, Lavender and Tuberose. Indian Journal of Pharmaceutical Sciences 71(2): 134-136.

Luis, C.J., Zairo, L.H., Pillar, P., Naira, D.G. (2002). A comparison between two brine shrimp assay to detect in vitro cytotoxicity in marine natural products. $B M C$ Biotechnology 2(17): 1-5.

Manasa, M., Poornima, G., Abhipsa, V., Rekha, C., Kekuda, P.T.R., Onkarappa, R., Mukunda, S. (2012). Antimicrobial and antioxidant potential of Streptomyces sp. RAMPP. 065 isolated from Kudremukh soil, Karnataka, India. Science, Technology \& Arts Research Journal 1(3):39-44.

Manivasagan, P., Gnanam, S., Sivakumar, K., Thangaradjou, T., Vijayalakshmi, S., Balasubramanian, T. (2009). Antimicrobial and cytotoxic activities of an actinobacteria (Streptomyces Sp. PM-32) isolated from an offshore sediments of the bay of Bengal in Tamilnadu. Advances in Biological Research 3(5-6): 231-236.

McLaughlin, J.L. (1991). Methods in plant biochemistry. In; Hostettmann K. Assay for bioactivity. Academic press.

Moncheva, P., Tishkov, S., Dimitrova, N., Chipeva, V., Nikolova, S.A., Bogatzevska, N. (2000-2002). Characteristics of soil actinomycetes from Antarctica. Journal of Culture Collections 3: 3-14.

Nalubega, R., Kabasa, J.D., Olila, D., Kateregga, J. (2011). Evaluation of antibacterial activity of selected ethnomedicinal plants for poultry in Masaka District, Uganda. Research Journal of Pharmacology 5(2): 18-21.

Nampoothiri, M.K., Ramkumar, B., Pandey, A. (2013). Western Ghats of India: Rich source of microbial diversity. Journal of Scientific and Industrial Research 72: 617-623.

Nanjwade, B., Chandrashekhara, S., Ali, M., Goudanavar, P.S., Manvi, F.V. (2010). Isolation and morphological characterization of antibiotic producing actinomycetes. Tropical Journal of Pharmaceutical Research 9(3):231-36.

Oskay, M., Tamer, A.U., Azeri, C. (2004). Antibacterial activity of some actinomycetes isolated from farming soils of Turkey. African Journal of Biotechnology 3(9): 441-446.

Pallavi, S., Manasa, M., Kambar, Y., Asha, M.M., Chaithra, M., Vivek, M.N., Swamy, S.H.C., Mesta, S.C., Onkarappa, R., Kekuda, P.T.R., Mallikarjun, N. (2013). AntiStaphylococcus aureus and Anti-yeast activity of Streptomyces species isolated from soil of Sahyadri Science College, Shivamogga, Karnataka. Asian Journal of Biomedical and Pharmaceutical Sciences 3(24): 07-11.

Parea, S., Patterson, T.F. (2002). Antifungal resistance in pathogenic fungi. Clinical Infectious Diseases 35:1073-80.

Priya, A.J., Sagadevan, E., Dhanalakshmi, P., Kumar, S.S., Karthikeyan, V., Arumugam, P. (2012). Detection of antioxidant and antimicrobial activities in marine actinomycetes isolated from Puducherry coastal region. Journal of Modern Biotechnology 1(2): 63-69.

Procopio, R.E.L., da Silvaa, I.R., Martinsa, M.K., de Azevedoa, J.L., de Araujob, J.M. (2012). Antibiotics
Sci. Technol. Arts Res. J., April-June 2015, 4(2): 164-180

produced by Streptomyces. The Brazilian Journal of Infectious Diseases 16(5): 466-471.

Re, R., Pellegrini, N., Proteggente, A., Pannala, A., Yang, M., Rice-Evans, C. (1999). Antioxidant activity applying an improved ABTS radical cation decolorization assay. Free Radical Biology and Medicine 26(9-10):1231-1237.

Rifaat, H.M. (2003). The biodiversity of Actinomycetes in the river Nile exhibiting antifungal activity. Journal of Mediterranean Ecology 4(3-4): 5-7.

Sahin, N., Ugur, A. (2003). Investigation of the antimicrobial activity of some Streptomyces isolates. Turkish Journal of Biology 27: 79-84.

Sharma, O.P., Bhat, T.K. (2009). DPPH antioxidant assay revisited. Food Chemistry 113(4): 1202-1205.

Sharmin, T., Rahman, M.A., Anisuzzaman, A.S.M., Islam, M.A. (2013). Antimicrobial and cytotoxic activities of secondary metabolites obtained from a novel species of Streptomyces. Bangladesh Pharmaceutical Journal 16(1): 15-19.

Shobha, K.S., Onkarappa, R. (2011). In vitro susceptibility of C. albicans and C. neoformens to potential metabolites from Streptomycetes. Indian Journal of Microbiology 51(4): 445-449.

Sowndhararajan, K., Kang, S.N. (2013). Evaluation of in vitro free radical scavenging potential of Streptomyces sp. AMS1 culture filtrate. Saudi Journal of Biological Sciences 20(3): 227-233.

Subathradevi, C., Devi, N.P., Jemimahnaine, S., Mohanasrinivasan, V. (2014). Antibacterial and antioxidant property of Streptomyces cinnamonensis VITNS1 isolated from Serkadu region, Vellore, Tamil Nadu, India. Anti-Infective Agents 12(2): 206-212.

Sultan, M.Z., Sathi, Z.S., Biswas, M.H.U., Choudury, M.A., Rahman, M.A.A., Gafur, M.A. (2002). In vitro toxicological studies of metabolites of Streptomyces species on brine shrimp. Journal of Medical Sciences 2(5-6): 240-242.

Taddei, A., Rodriguez, M.J., Marquez-Vilchez, E., Castelli, C. (2006). Isolation and identification of Streptomyces spp. From Venezuelan soils: Morphological and biochemical studies. I. Microbiological Research 161: 222-231.

Taechowisan, T., Lu, C., Shen, Y., Lumyong, S. (2005). Secondary metabolites from endophytic Streptomyces aureofaciens CUMAc130 and antifungal activity. Microbiology 151: 1691-1695.

Teow, C.C., Truong, V., McFeeters, R.F., Thompson, R.L., Pecota, K.V., Yencho, G.C. (2007). Antioxidant activities, phenolic and $\beta$-carotene contents of sweet potato genotypes with varying flesh colours. Food Chemistry 103: 829-838.

Xu, L.H., Li, Q.R., Jiang, C.L. (1996). Diversity of Soil Actinomycetes in Yunnan, China. Applied and Environmental Microbiology 62(1): 244-248.

Yaacob, N.S., Hamzah, N., Kamal, N.N.N.M., Abidin, S.A.Z., Lai, C.S., Navaratnam, V., Norazmi, M.N. (2010). Anticancer activity of a sub-fraction of dichloromethane extract of Strobilanthes crispus on human breast and prostate cancer cells in vitro. BMC Complementary Alternative Medicine 10: 42.

Yadav, J., Shrestha, U.T., Tiwari, K.B., Sahukhal, G.S., Agarwal, V.P. (2008). Streptomycin-like antibiotic from Streptomyces spp. isolated from Mount Everest base camp. Nepal Journal of Science and Technology 9:73-77.

Zhong, K., Gao, X., Xu, Z., Gao, H., Fan, S., Yamaguchi, I., Li, L., Chen, R. (2011). Antioxidant activity of a novel Streptomyces strain Eri12 isolated from the rhizosphere of Rhizoma curcumae Longae. Current Research in Bacteriology 4(2): 63-72. 